Notre Dame Journal of Formal Logic

Volume 48, Number 1, 2007

\title{
Computability of Homogeneous Models
}

\author{
Karen Lange and Robert I. Soare
}

\begin{abstract}
In the last five years there have been a number of results about the computable content of the prime, saturated, or homogeneous models of a complete decidable $(\mathrm{CD})$ theory $T$ in the spirit of Vaught's "Denumerable models of complete theories" combined with computability methods for (Turing) degrees $\mathbf{d} \leq \mathbf{0}^{\prime}$. First we recast older results by Goncharov, Peretyat'kin, and Millar in a more modern framework which we then apply. Then we survey recent results by Lange, "The degree spectra of homogeneous models," which generalize the older results and which include positive results on when a certain homogeneous model $\mathcal{A}$ of $T$ has an isomorphic copy of a given Turing degree. We then survey Lange's "A characterization of the 0-basis homogeneous bounding degrees" for negative results about when $\mathcal{A}$ does not have such copies, generalizing negative results by Goncharov, Peretyat'kin, and Millar. Finally, we explain recent results by Csima, Harizanov, Hirschfeldt, and Soare, "Bounding homogeneous models," about degrees $\mathbf{d}$ that are homogeneous bounding and explain their relation to the PA degrees (the degrees of complete extensions of Peano Arithmetic).
\end{abstract}

\section{Introduction}

Vaught [34] studied countable models of a countable complete theory $T$ and introduced the notions of prime, homogeneous, and saturated models, defined using the types in the Stone space $S(T)$. These models, which we call Vaughtian models, have played an important role both in classical model theory and in computable model theory. (We shall give all formal definitions in later sections.)

Convention 1.1 (Countability Convention) Unless otherwise stated all theories $T$ here will be consistent, countable, and complete, having infinite models, as in Vaught [34], and all models $\mathcal{A}$ of $T$ will be countable, as in Vaught [34], Sections $1-4$.

Received May 11, 2006; accepted December 15, 2006; printed February 26, 2007 2000 Mathematics Subject Classification: Primary, 03D35, 03D30, 03D80

Keywords: prime, saturated, homogeneous, model, computable model theory, compatability theory

(C)2007 University of Notre Dame 
1.1 Early results on computability of Vaughtian models During the 1970s, researchers combined computability theory with these Vaughtian models by considering a complete decidable (CD) theory $T$ and examining the computable content of these models and of the Vaught-style constructions. For a (Turing) degree d, a model $\mathcal{A}$ of $T$ is $\mathbf{d}$-decidable if its elementary diagram $D^{e}(\mathcal{A})$ is $\mathbf{d}$-computable and $\mathcal{A}$ is $\mathbf{d}$-computable if its atomic diagram $D(\mathscr{A})$ is $\mathbf{d}$-computable. (Note that we use the same terminology for degrees and sets.) Harrington [9] and independently Goncharov-Nurtazin [8] found a criterion under which a complete atomic decidable (CAD) theory $T$ has a decidable prime model. Morley [25], and independently Millar [24], gave a criterion under which a CD theory $T$ with types all computable (TAC) has a decidable saturated model. Goncharov [7] and independently Peretyat'kin [27] gave a criterion expressed in terms of types for the decidability of a homogeneous model $\mathcal{A}$ of a CD theory. In later sections we shall review these and other older results and recast some in a modern format suitable for application to recent results.

1.2 Other degrees beside 0 and $0^{\prime} \quad$ Generally, the results of twenty or thirty years ago drew a sharp dichotomy between $\mathbf{0}$ and $\mathbf{0}^{\prime}$. They often showed that a CD theory $T$ with certain hypotheses does have a certain Vaughtian model (prime, saturated, homogeneous) which is $\mathbf{0}^{\prime}$-decidable but that it does not always have a $\mathbf{0}$-decidable such model.

After Post's Problem [28] was solved, a great deal of attention in computability theory has been given to degrees $\mathbf{d}$ such that $\mathbf{0}<\mathbf{d}<\mathbf{0}^{\prime}$, to degrees of complete extensions of Peano Arithmetic (PA degrees), and to other classes of degrees. For example, Martin's beautiful theorem [21] related the high computably enumerable (c.e.) degrees to the degrees of maximal sets. Likewise, results of the last five years link Vaughtian models to these rich classes of degrees, which sharpen our understanding of both the model theory and the computability theory.

A degree $\mathbf{d} \leq \mathbf{0}^{\prime}$ is $h i g h_{n}$ if $\mathbf{d}^{(n)}=\mathbf{0}^{(n+1)}$, the highest possible value. A degree $\mathbf{d}$ is low $w_{n}$ if $\mathbf{d}^{(n)}=\mathbf{0}^{(n)}$, the lowest possible value, and $\mathbf{d}$ is high if it is high $h_{1}$ and

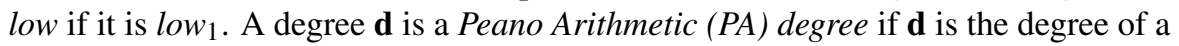
complete extension of the effectively axiomatized theory of Peano Arithmetic.

1.3 Recent results on prime models Let $T$ be a complete atomic theory. Vaught proved that $T$ has a prime model. (See Vaught [34], Theorem 2.2.1 and Theorem 3.5; see also Marker [20], Theorem 4.2.10.) Decades ago it was noted that if $T$ is a complete atomic decidable $(\mathrm{CAD})$ theory, then Vaught's proof produces a $\mathbf{0}^{\prime}$-decidable prime model because the question of whether a formula $\theta(\bar{x})$ splits is a $\Pi_{1}$-property. Hence, a $\mathbf{0}^{\prime}$-oracle can extend any formula to an atom and can produce a prime model.

Moving to a finer classification of the degree spectrum of prime models, Csima [3] considerably strengthened this result by proving that every CAD theory $T$ has a prime model whose degree is 1-generic and hence has low degree. Csima also showed that if $T$ has types all computable (TAC) then every nonzero degree $\mathbf{d} \leq \mathbf{0}^{\prime}$ is realized as the degree of a prime model. By a clever and very short proof, Hirschfeldt [12] extended this to all nonzero degrees. Csima has other results about degrees of prime models below $\mathbf{0}^{\prime}$ including halves of minimal pairs, avoiding cones, and results which suggest a profusion of such degrees, although their exact classification in the non-TAC case remains open. 
Epstein [6] has been exploring the properties of computably enumerable degrees of prime and homogeneous models. Epstein has improved Csima's low prime model theorem to show that any CAD theory $T$ has a prime model of low c.e. degree and that many of Csima's other results on degrees of prime models can be sharpened to c.e. degrees. She has also explored the extent to which results about degrees of homogeneous models can be sharpened to c.e. degrees.

These results have to do with the spectrum of degrees of prime models for a given CAD theory $T$. Now we define a degree $\mathbf{d}$ to be prime bounding if every CAD theory $T$ has a d-decidable prime model. Csima, Hirschfeldt, Knight, and Soare [5] showed that a $\Delta_{2}^{0}$ degree $\mathbf{d}$ is prime bounding if and only if it is nonlow 2 $\left(\mathbf{d}^{\prime \prime}>\mathbf{0}^{\prime \prime}\right)$. That paper contains eight properties about a bounding set $X$, some computability theoretic properties ( $X$ nonlow $_{2}, X$ can compute an escape function), and some purely algebraic or topological ( $X$ can compute a path through a certain $\Delta_{2}$ dense open set, $X$ can omit certain types, $X$ can compute certain reduced $p$-groups).

Surprisingly all eight properties were proved equivalent for $X \leq \mathbf{0}^{\prime}$. However, without the hypothesis $X \leq \mathbf{0}^{\prime}$ the situation is much more complicated. In this general framework of $X \not \leq \mathbf{0}^{\prime}$, Conidis [2] has used forcing arguments and priority arguments to determine the implications and nonimplications between these properties. Separating some of these properties is extremely complicated, but has a general mathematical flavor since these properties are found in many branches of mathematics.

1.4 Recent results on saturated models For saturated models we consider a CD theory $T$ with types all computable (TAC) because any noncomputable type must be realized in any saturated model $\mathcal{A}$ of $T$ and will therefore raise the degree of the elementary diagram $D^{e}(\mathcal{A})$, perhaps even out of the arithmetic hierarchy. It is easy to see that Vaught's proof shows that $T$ has a $\mathbf{0}^{\prime}$-decidable model. Morley and Millar independently showed that $T$ has a $\mathbf{0}$-decidable saturated model if the types of $S(T)$ are uniformly computable, but Millar produced a CD + TAC theory $T$ with no $\mathbf{0}$-decidable saturated model.

A degree $\mathbf{d}$ is saturated bounding if every CD theory with TAC has a d-decidable saturated model. Macintyre and Marker [19] proved that every PA degree is saturated bounding. Harris [10] proved that this result also follows from work of Jockusch [13], as does the fact that every high degree is saturated bounding.

In the other direction, Harris [11] first proved a new and very elegant characterization of the $\operatorname{low}_{n}$ degrees in terms of escape functions. Using this characterization, he established in [10] that no c.e. degree $\mathbf{d}$ that is low or even $\operatorname{low}_{n}$ can be saturated bounding.

1.5 Copies of a homogeneous model: Positive results Morley [25] helped initiate the study of when a homogeneous model $\mathcal{A}$ of a complete decidable theory $T$ has a decidable copy $\mathcal{B}$. Clearly, there must be a uniformly computable enumeration $X=\left\{p_{j}\right\}_{j \in \omega}$ of the set of types $\mathbb{T}(\mathcal{A})$ realized in $\mathcal{A}$. We call $X$ a $\mathbf{0}$-basis for $\mathbb{T}(\mathcal{A})$. (There could be many such $\mathbf{0}$-bases, some more favorable for our purposes than others.) Morley's Question 3.7, which heavily influenced all these results, was whether every homogeneous model $\mathcal{A}$ with a $\mathbf{0}$-basis $X$ has a decidable copy.

Goncharov [7] and Peretyat'kin [27] showed that the computability of an additional function on the $\mathbf{0}$-basis $X$ is required, an effective extension function (EEF), 
a computable function $f$ for extending a type $p \in \mathbb{T}(\mathcal{A})$ to a certain other type $q \in \mathbb{T}(\mathcal{A})$ (see Definition 4.2). Both in the positive and negative direction, a more useful property is Goncharov's notion of a monotone extension function (MEF) (Definition 4.6), a computable function $\widehat{g}$ which gives a monotone $\Delta_{2}^{0}$ approximation to an extension function $g(x)=\lim _{s} \widehat{g}(x, s)$. In Section 4.4 we explore the relation of the EEF to MEF.

Fix a homogeneous model $\mathcal{A}$ of a $\mathrm{CD}$ theory $T$ for which $\mathbb{T}(\mathcal{A})$ has a $\mathbf{0}$-basis but no EEF (and hence no decidable copy). Lange [18] studied the degrees of isomorphic copies of $\mathcal{A}$ and proved three results which are positive in that they show the existence of copies of $\mathcal{A}$ of a certain degree. Some results use the EEF/MEF functions relativized to the appropriate oracles. First, Lange used a forcing argument to show that $\mathcal{A}$ always has a copy of 1 -generic degree and, therefore, of low degree. This holds even with the weaker hypothesis of a $\mathbf{0}^{\prime}$-basis (Definition 5.1) in place of a $\mathbf{0}$-basis and implies the Csima low basis theorem for prime models [3] mentioned above. Second, she showed that if $\mathbf{d} \leq \mathbf{0}^{\prime}$ is not low $2\left(\mathbf{d}^{\prime \prime}>\mathbf{0}^{\prime \prime}\right)$ then $\mathcal{A}$ has a copy of degree d. Note that we always work with the hypothesis that all types realized in $\mathcal{A}$ are computable, that is, $\mathbb{T}(\mathcal{A})$ satisfies TAC, but so far we have not examined those types $p$ not realized in $\mathcal{A}$, that is, $p \in S(T)-\mathbb{T}(\mathcal{A})$. It might seem that these will not affect the degrees of copies of $\mathcal{A}$, but they have a strong effect. For her third result in the positive direction, Lange proved that if all types in $S(T)$ are computable, then every nonzero degree is the degree of some copy of $\mathcal{A}$. This uses a device by Hirschfeldt [12] to prove the corresponding result for prime models, but neither theorem implies the other.

1.6 Copies of a homogeneous model: Negative results In the other direction, Goncharov [7], Peretyat'kin [27], and Millar [23] negatively answered Morley's Question 3.7 by constructing a CD theory $T$ and a homogeneous model $\mathscr{A}$ of $T$ with a $\mathbf{0}$-basis but no decidable copy. To better understand the results we present a slightly different approach. By the EEF Theorem 4.3 mentioned above and its relation to the MEF Theorem 4.8 described in Section 4.4, this result is equivalent to our constructing a homogeneous model $\mathcal{A}$ and a $\mathbf{0}$-basis $X$ such that $X$ has no MEF. This makes it easier to build a homogeneous model $\mathcal{A}$ and some $\mathbf{0}$-basis $X$ for $\mathbb{T}(\mathcal{A})$ and to diagonalize over all possible MEF functions for $X$, thereby ensuring that $\mathcal{A}$ has no decidable copy.

Lange [17] then obtained a much stronger negative result by constructing a CD theory $T$, a homogeneous model $\mathcal{A}$ of $T$, and a $\mathbf{0}$-basis $X$ for $\mathcal{A}$ such that $\mathcal{A}$ has no low $_{2}$ copy. Hence, Lange's nonlow 2 homogeneous bounding result in the preceding section is best possible.

1.7 Homogeneous bounding degrees So far we have been considering the degrees of copies of a fixed model $\mathcal{A}$ of a single CD theory $T$. Now, by analogy with the prime model case, let us define a degree $\mathbf{d}$ to be homogeneous bounding if for every $\mathrm{CD}$ theory $T$ there is a d-decidable homogeneous model $\mathcal{A}$ of $T$. A degree $\mathbf{d}$ is a PA degree if $\mathbf{d}$ is the degree of a complete extension of Peano Arithmetic (PA). There are a number of equivalent definitions, one of which is equivalent to Weak König's Lemma about the existence of an infinite path $f \leq \mathbf{d}$ through an infinite computable binary tree $\mathcal{T}$. 
There are several different proofs that every PA degree is homogeneous bounding, one of which we shall sketch in Section 7. In addition, the paper by Csima, Harizanov, Hirschfeldt, and Soare [30] proved the converse that every homogeneous bounding degree is a PA degree. We give a brief idea of the proof which requires constructing a CD theory $T$ such that any homogeneous model is able to separate a pair of effectively inseparable c.e. sets, another equivalent property of PA degrees. The proof gives some insight into the property of a model being homogeneous.

1.8 History and terminology of Vaughtian models We define Vaughtian models to be countable models of a complete theory $T$ which are prime, saturated, or homogeneous. This is the background. Vaught [34] cites Ryll-Nardzewski [29] who proved that a complete countable theory $T$ is $\omega$-categorical if and only if the Lindenbaum algebra $B_{n}(T)$ is finite for every $n$. In the sufficiency direction Ryll-Nardzewski proved that if $B_{n}(T)$ is finite for every $n$ and $\mathcal{A}$ and $\mathscr{B}$ are models of $T$ then the atoms of $B_{n}(T)$ can be used to produce the isomorphism from $\mathcal{A}$ to $\mathscr{B}$. This is close to Vaught's later proof that if $\mathcal{A}$ and $\mathscr{B}$ are countable and atomic then they are isomorphic. Ehrenfeucht had invented the omitting types argument and used it to prove the necessary direction of Ryll-Nardzewski's theorem. Ryll-Nardzewski does not mention the notion of prime models, which had been studied by Robinson for ordinary (nonelementary) embeddings. He, however, had been studying model-complete theories where these coincide. All these elements on prime models, countable atomic models, omitting types, atoms and atomless elements of $B_{n}(T)$, atomistic (atomic) algebras $B_{n}(T)$, were brought together by Vaught first in an abstract [32], and then in his main paper [34].

Vaught ([34], p. 1) boldly introduced the notion of a (countable) model $\mathcal{A}$ being homogeneous before prime or saturated (although most model theory books do the reverse). He defined $\mathcal{A}$ to be homogeneous as in Definition 2.7 if every finite partial map can be extended to an automorphism of $\mathcal{A}$. This is apparently the first time this notion has appeared in the literature. Previously there had been several papers, such as Jonsson [15], [14], Vaught [33], [31], and Morley-Vaught [26], on the term "homogeneous universal" systems, but this always had meant "saturated," never simply "homogeneous," and had always referred to uncountable models of large cardinality. Vaught [32] had declared a "saturated" model to mean a countable which is an elementary extension of every countable model of $T$. Thus, Vaught [32] and [34] was the first to study homogeneous or countable saturated models of $T$.

We conclude that it is fair to say that, in the countable case, Vaught introduced the now standard concepts of prime (and atomic), saturated, and homogeneous models. For this reason, we call prime, saturated, and homogeneous countable models "Vaughtian models."

\section{Preliminary Results and Notation}

Let $T$ be a (consistent) theory in a computable language $\mathcal{L}$. We replace $\mathcal{L}$ if necessary by $\mathscr{L}_{c}$, an expansion obtained by adding an infinite set of new (Henkin) constants $C=\left\{c_{j}\right\}_{j \geq 1}$ and letting $T_{c}$ be the theory in $\mathcal{L}_{c}$ consisting of $T$ together with usual Henkin axioms of the form $(\exists x) \theta(x) \rightarrow \theta\left(c_{j}\right)$ for some $c_{j}$. Note that $T_{c}$ is a conservative extension of $T$ and has the same Turing degree. Any complete extension of $T_{c}$ in $\mathscr{L}_{c}$ corresponds to the elementary diagram $D^{e}(\mathcal{A})$ of the corresponding canonical Henkin model. (See Marker [20], p. 39.) By Lindenbaum's Lemma we can 
always find a complete extension of $T_{c}$, but if we want the corresponding canonical model to have special properties such as being prime, saturated, or homogeneous, we must control the types realized in $\mathcal{A}$.

2.1 Trees and $\Pi_{\mathbf{1}}^{\mathbf{0}}$ classes The Vaught theorems and our computability theoretic results make considerable use of trees. Types will be viewed as paths on trees, and all the familiar terminology and results about types will be developed first for trees. We begin the tree definitions here and continue with more properties in Definition 2.5 on atomic trees and atomic theories and models where we define atoms and isolated (principal) types.

\section{Definition 2.1 (Trees, Part 1)}

(i) A tree $\mathcal{T} \subseteq 2^{<\omega}$ is a subset of $2^{<\omega}$ closed under initial segment; that is, $\tau \subset \sigma \in \mathcal{T}$ implies $\tau \in \mathcal{T}$. Define the set of (infinite) paths,

$$
[\mathcal{T}]=\left\{f: f \in 2^{\omega} \quad \& \quad(\forall x)[f\lceil x \in \mathcal{T}]\} .\right.
$$

(ii) Cantor space is $2^{\omega}$ with the following topology. (In the context of trees of types with the same topology this is called the Stone Space.) Let $\sigma \subset f$ denote that $f$ extends $\sigma$. For every $\sigma \in 2^{<\omega}$ define the basic open set,

$$
u_{\sigma}=\left\{f: f \in 2^{\omega} \& \sigma \subset f\right\} .
$$

(iii) A class $\mathcal{C} \subset 2^{\omega}$ is closed if $2^{\omega}-\mathcal{C}$ is open or equivalently if $\mathcal{C}=[\mathcal{T}]$ for some tree as in (1).

(iv) If $\mathcal{C}=[\mathcal{T}]$ for some computable tree $\mathcal{T}$, then $\mathcal{C}$ is effectively closed and is called a $\Pi_{1}^{0}$-class, a very important concept.

(v) A tree $\mathcal{T}$ is extendible if every node $\sigma$ can be extended to an infinite path in $\mathcal{T}$; that is,

$$
(\forall \sigma \in \mathcal{T})(\exists f \supset \sigma)[f \in[\mathcal{T}]] .
$$

A $\Pi_{1}^{0}$-class $\mathcal{C}$ is extendible if $\mathcal{C}=[\mathcal{T}]$ for some extendible tree $\mathcal{T}$, and $\mathcal{C}$ is nonextendible otherwise.

2.2 The Lindenbaum algebra $B_{n}(T)$ of formulas From now on let $\mathcal{L}$ be a computable language, meaning we can effectively determine the arity of the function, relation, and constant symbols.

\section{Definition 2.2 (Lindenbaum Algebra)}

(i) Let $F_{n}(\mathscr{L})$ be set of the formulas $\theta\left(x_{0}, \ldots, x_{n-1}\right)$ of $\mathscr{L}$ with free variables included in $x_{0}, \ldots, x_{n-1}$. Let $F(\mathscr{L})=\cup_{n \geq 0} F_{n}(\mathscr{L})$.

(ii) The equivalence class of $\theta(\bar{x}) \in F_{n}(\mathscr{L})$ under $T$-provability $\vdash_{T}$ is

$$
\theta^{*}(\bar{x})=\left\{\gamma(\bar{x}): \vdash_{T}(\forall \bar{x})[\theta(\bar{x}) \leftrightarrow \gamma(\bar{x})]\right\},
$$

and the Lindenbaum algebra $B_{n}(T)$ consists of these equivalence classes under the induced operations. We often identify $\theta(\bar{x})$ and its equivalence class $\theta^{*}(\bar{x})$.

(iii) Let $\left\{\theta_{i}(\bar{x})\right\}_{i \in \omega}$ be an effective listing of $F_{n}(\mathscr{L})$. For every string $\alpha \in 2^{<\omega}$ define

$$
\left.\left[\theta_{\alpha}(\bar{x})\right]=\bigwedge_{i}^{\alpha(i)}(\bar{x}): i<|\alpha|\right\}
$$

where $\theta^{1}=\theta$ and $\theta^{0}=\neg \theta$. 


\subsection{The Stone space $S_{n}(T)$ as paths in the tree $\mathcal{T}_{n}(T)$}

Definition 2.3 Let $T$ be a complete $\mathcal{L}$-theory.

(i) A formula $\theta(\bar{x}) \in F_{n}(\mathcal{L})$ is consistent with $T$ if $T \cup(\exists \bar{x}) \theta(\bar{x})$ is consistent, that is, if $T \vdash(\exists \bar{x}) \theta(\bar{x})$, because $T$ is complete. Let $F_{n}(T)$ be the subset of $F_{n}(\mathcal{L})$ consisting of all formulas consistent with $T$.

(ii) Define the tree of $n$-ary formulas consistent with $T$,

$$
\mathcal{T}_{n}(T)=\left\{\theta_{\alpha}(\bar{x}): \alpha \in 2^{<\omega} \quad \& \quad(\exists \bar{x}) \theta_{\alpha}(\bar{x}) \in T\right\} .
$$

If $\alpha \subset \beta$, then we say that $\beta$ lies below $\alpha$, that $\theta_{\beta}$ extends $\theta_{\alpha}$ and contains more information. (Note that the equivalence classes $\left\{\left[\theta_{\alpha}\right]: \alpha \in \mathcal{T}_{n}(T)\right\}$ generate the Lindenbaum algebra $B_{n}(T)$ under the Boolean operations.)

(iii) We regard $\alpha$ as an index of $\theta_{\alpha}$. Define the tree of indices,

$$
\widehat{\mathcal{T}}_{n}(T)=\left\{\alpha: \theta_{\alpha} \in \mathcal{T}_{n}(T)\right\}
$$

The trees $\mathcal{T}_{n}(T)$ and $\widehat{\mathcal{T}}_{n}(T)$ are effectively isomorphic but $\widehat{\mathcal{T}}_{n}(T) \subseteq 2^{<\omega}$ and is notationally simpler. Hence, any definitions or results on trees $\widehat{\mathcal{T}} \subset 2^{<\omega}$ automatically carry over to $\mathcal{T}_{n}(T)$. We mostly suppress explicit mention of $\widehat{\mathcal{T}}_{n}(T)$ and simply identify $\alpha$ and $\theta_{\alpha}(\bar{x})$.

\section{Definition 2.4 (Types and the Stone Space)}

(i) An $n$-type of $T$ is a maximal consistent subset $p$ of formulas of $F_{n}(T)$. There is a 1-1 correspondence between paths $f \in\left[\widehat{\mathcal{T}}_{n}(T)\right] \subset 2^{\omega}$ and the corresponding types $p_{f} \in\left[\mathcal{T}_{n}(T)\right]$ where

$$
p_{f}=\left\{\theta_{\alpha}(\bar{x}): \theta_{\alpha}(\bar{x}) \in \mathcal{T}_{n}(T) \quad \& \quad \alpha \subset f\right\} .
$$

(ii) $S_{n}(T)$ is the set of all $n$-types of $T$, with the usual topology as in Definition 2.1, and it is also called the Stone Space (i.e., the dual space of the Boolean algebra $\left.B_{n}(T)\right)$. The clopen sets of the Cantor space are given by

$$
u_{\alpha}=\{f: \alpha \subset f\}
$$

(iii) Define $S(T)=\cup_{n \geq 1} S_{n}(T)$. (We can also regard $S(T)$ as homeomorphic to a subset of $2^{\omega}$ as follows. Build a tree $\mathcal{T} \subset 2^{<\omega}$ by putting $1^{n-0}$ on $\mathcal{T}$ and then putting an isomorphic copy of $\widehat{\mathcal{T}}_{n}(T)$ above it on $\mathcal{T}$.)

(iv) Hence, $S_{0}(T)$ is the set of complete extensions of $T$, that is, 0-types of $T$. Since we assume $T$ to be complete theory, $S_{0}(T)$ consists of a single path. However, $S_{0}\left(T_{c}\right)$ usually consists of more than one path. Since $T_{c}$ is Henkinized, every such path determines a Henkin model $\mathcal{A}$ of $T_{c}$.

2.4 Atomic trees and principal types We continue Definition 2.1 with properties of trees which will also apply to types.

Definition 2.5 (Trees, Part 2) Let $\mathcal{T} \subseteq 2^{<\omega}$ be an extendible tree.

(i) Nodes $\beta, \gamma$ are incomparable, written $\alpha \mid \gamma$, if $(\exists k)[\beta(k) \downarrow \neq \gamma(k)]$.

(ii) Nodes $\beta, \gamma \in \mathcal{T}$ split node $\alpha$ on $\mathcal{T}$ if $\alpha \subset \beta, \alpha \subset \gamma$, and $\beta \mid \gamma$.

(iii) Node $\alpha \in \mathcal{T}$ is an atom if no extensions split $\alpha$ on $\mathcal{T}$. If $\alpha$ is an atom then the unique extension of $f \supset \alpha$ on $\mathcal{T}$ is an isolated (principal) path of [ $\mathcal{T}], \alpha$ is a 
generator of $f$, and we say $\alpha$ isolates $f$. Note that $f$ is isolated in the topological sense by the basic open set $U_{\alpha}$ because

$$
u_{\alpha} \cap[\mathcal{T}]=\{f\} .
$$

(iv) For a complete theory $T$ we define the set of principal types,

$$
S^{P}(T)=\{p: p \text { is a principal type of } S(T)\} .
$$

(v) $\mathcal{T}$ is atomic if for every $\beta \in \mathcal{T}$ there is an atom $\alpha \supseteq \beta$ with $\alpha \in \mathcal{T}$, or equivalently if the isolated points of $[\mathcal{T}]$ are dense in $[\mathcal{T}]$.

(vi) A complete theory $T$ is atomic if tree $\mathcal{T}_{n}(T)$ is atomic for every $n \geq 1$.

\subsection{The type spectrum $\mathbb{T}(\mathcal{A})$ of a model $\mathcal{A}$}

Definition 2.6 Let $T$ be a theory and $\mathcal{A}$ a model of $T$.

(i) An $n$-tuple $\bar{a} \in A$ realizes an $n$-type $p(\bar{x}) \in S_{n}(T)$ if $\mathcal{A} \models \theta(\bar{a})$ for all $\theta(\bar{x}) \in p(\bar{x})$. In this case we say that $\mathcal{A}$ realizes $p$ via $\bar{a}$.

(ii) Define the type spectrum of $\mathcal{A}$

$$
\mathbb{T}(\mathcal{A})=\{p: p \in S(T) \quad \& \quad \mathcal{A} \text { realizes } p\}, \quad \text { and }
$$

(iii) $\mathbb{T}_{n}(\mathcal{A})=\mathbb{T}(\mathcal{A}) \cap S_{n}(T)$, the $n$-types realized in $\mathcal{A}$.

In early papers some authors in computable model theory had used $S(\mathcal{A})$ in place of $\mathbb{T}(\mathcal{A})$. However, this conflicts with the standard usage in ordinary model theory where Marker ([20], p. 115) defines $S_{n}^{\mathcal{A}}(Y)$ to be the set of $n$-types in the theory $\operatorname{Th}_{Y}(\mathcal{A})$ of $\mathcal{A}$ for some $Y \subseteq|\mathcal{A}|$. The use of $\mathbb{T}_{n}(\mathcal{A})$ is different from Marker's $S_{n}^{\mathcal{A}}(Y)$ because (1) we consider only pure types in the original language $\mathcal{L}=\mathscr{L}(T)$ and do not allow any extra names $Y \subseteq|\mathcal{A}|$ to be added; (2) we consider only those types actually realized in $\mathcal{A}$ not merely those consistent with $\mathrm{Th}_{Y}(\mathcal{A})$. Marker has no notation for our $\mathbb{T}_{n}(\mathcal{A})$. The use of $\mathbb{T}_{n}(\mathcal{A})$ rather than $f_{n}^{\mathcal{A}}(Y)$ is particularly useful for studying homogeneous models as we now see.

2.6 The uniqueness theorem for homogeneous models Early in the history of model theory Vaught [34] studied homogeneous models. Let $\mathcal{A} \equiv \mathscr{B}$ denote elementary equivalence of $\mathcal{A}$ and $\mathscr{B}, \mathcal{A} \cong \mathscr{B}$ denote isomorphism of $\mathcal{A}$ and $\mathcal{B}$, and Aut $\mathcal{A}$ denote the group of automorphisms of $\mathcal{A}$.

Definition 2.7 A model $\mathcal{A} \models T$ is homogeneous if for all $n$-tuples $\bar{a}$ and $\bar{b}$,

$$
(\mathcal{A}, \bar{a}) \equiv(\mathcal{A}, \bar{b}) \quad \Longrightarrow \quad(\exists \Phi \in \text { Aut } \mathcal{A})[\Phi(\overline{\mathrm{a}})=\overline{\mathrm{b}}] .
$$

One of the most pleasant properties of homogeneous models is the following result which demonstrates the usefulness of the notion $\mathbb{T}(\mathcal{A})$. (See Marker [20], Theorem 4.3.23 for the general case and Theorem 4.2.15 for the countable case, which is all we need in this paper. Apparently, the general case first appeared in Keisler and Morley [16].)

Theorem 2.8 (Uniqueness of Homogeneous Models) Given a countable complete theory $T$ and homogeneous models $\mathcal{A}$ and $\mathscr{B}$ of $T$ of the same cardinality

$$
\mathbb{T}(\mathcal{A})=\mathbb{T}(\mathscr{B}) \quad \Longrightarrow \quad \mathcal{A} \cong \mathscr{B} .
$$


Hence, for an arbitrary homogeneous model $\mathcal{A}$ (and therefore for prime and saturated models, which are necessarily homogeneous) the isomorphism type of $\mathcal{A}$ is completely determined by the types realized in the model. This will be very useful for passing from a homogeneous model $\mathcal{A}$ to an isomorphic copy $\mathscr{B}$ which is d-decidable for an appropriate degree d. We simply construct a d-decidable homogeneous model $\mathcal{B}$ with $\mathbb{T}(\mathscr{B})=\mathbb{T}(\mathcal{A})$.

2.7 Prime and saturated models The terminology of homogeneous models appeared in Jonsson [14] and Vaught [33], [31], and [34], although the universal homogeneous models studied there were later seen to be equivalent to saturated. The terminology of prime and saturated models appeared in Vaught [32] and [34].

Definition 2.9 Let $T$ be a complete theory.

(i) A model $\mathscr{A}$ of $T$ is prime if $\mathscr{A}$ can be elementarily embedded in any other model $\mathcal{B}$ of $T$.

(ii) $\mathcal{A}$ is weakly saturated if $\mathbb{T}(\mathcal{A})=S(T)$, and a countable model $\mathcal{A}$ is (countably) saturated if $\mathcal{A}$ realizes every type defined over a finite set $F \subseteq A$.

Vaught [34] proved that a model $\mathcal{A}$ is prime if and only if $\mathcal{A}$ is countable and atomic, that is, realizes only principal types. This is often taken as the defining property of prime models. When we write "prime" we shall always mean "countable and atomic." Since we are dealing in this paper exclusively with countable homogeneous models of a complete theory $T$ we note the following.

Remark 2.10 (Vaught [34]) Let $T$ be a complete theory and $\mathcal{A}$ be a countable homogeneous model of $T$.

(i) $\mathcal{A}$ is prime iff $\mathbb{T}(\mathcal{A})=S^{P}(T)$, the principal types of $T$.

(ii) $\mathcal{A}$ is saturated iff $\mathcal{A}$ is weakly saturated iff $\mathbb{T}(A)=S(T)$.

Proof Part (i) follows because $\mathbb{T}(\mathcal{A}) \subseteq S^{P}(T)$ by Vaught's prime model theorem above. Furthermore, in a complete theory $T$ any principal type $p$ is realized in any model $\mathcal{A}$ of $T$. Hence, $\mathbb{T}(\mathcal{A})=S^{P}(T)$.

Part (ii) follows because if $\mathcal{A}$ is countable and weakly saturated then $S(T)$ is countable. Thus, $T$ has a saturated model $\mathscr{B}$ which is homogeneous by Vaught [34]. But then $\mathbb{T}(\mathscr{A})=\mathbb{T}(\mathscr{B})=S(T)$. Hence, $\mathscr{A} \cong \mathscr{B}$ by the Homogeneous Uniqueness Theorem 2.8.

If $\mathcal{A}$ is a homogeneous model of a complete theory $T$, then we have

$$
S^{P}(T) \subseteq \mathbb{T}(\mathcal{A}) \subseteq S(T) .
$$

If $\mathbb{T}(\mathcal{A})$ coincides with the left endpoint $S^{P}(T)$, then $\mathcal{A}$ is a prime model. If $\mathbb{T}(\mathcal{A})$ coincides with the right-hand endpoint $S(T)$, then $\mathcal{A}$ is a saturated model. Otherwise, $\mathbb{T}(\mathcal{A})$ will take an intermediate value and the isomorphism type of $\mathcal{A}$ will be completely determined by the type spectrum $\mathbb{T}(\mathcal{A})$. This will be helpful later in constructing isomorphic copies of $\mathcal{A}$ while controlling the degree.

This situation is roughly analogous to the case of the countable models of a theory $T$ that is $\omega_{1}$-categorical but not $\omega$-categorical. By the Baldwin-Lachlan theorem [1] its countable models form an elementary chain of length $\omega+1$,

$$
\mathcal{M}_{0} \prec \mathcal{M}_{1} \prec \cdots \quad \prec \mathcal{M}_{\omega}
$$


where $\mathcal{M}_{0}$ is the prime model and $\mathcal{M}_{\omega}$ is the countable saturated model of $T$. For example, if $T=A C F_{0}$, the theory of algebraically closed fields, then the countable models $\mathcal{M}_{i}$ are all homogeneous, a special case of (3).

In the case of countable homogeneous models of $T$ we may not have a countable saturated model or even a prime model. However, if we do have a countable saturated model, then we have a prime model so that the homogeneous models determined by (3) form a partially ordered set

$$
\mathcal{A}_{0} \subset \mathcal{A}_{\alpha} \subset \mathcal{A}_{\beta} \subset \cdots \subset \mathcal{A}_{\omega}
$$

with $\mathcal{A}_{0}$ the prime model contained in every member $\mathcal{A}_{\alpha}, \mathcal{A}_{\omega}$ the countable saturated model containing every $\mathcal{A}_{\alpha}$, and the relationship of inclusion $\mathcal{A}_{\alpha} \subset \mathcal{A}_{\beta}$ or incomparability $\mathcal{A}_{\beta} \mid \mathcal{A}_{\gamma}$ among the homogeneous models being determined entirely by the inclusion relation of the types realized in them.

\subsection{Realizing a type spectrum $\mathcal{C}$ in a homogeneous $\mathcal{A}$}

Definition 2.11 Let $T$ be a complete theory. For an $n$-type $p(\bar{x}) \in S_{n}(T)$ and an $(n+1)$-ary formula $\theta(\bar{x}, y) \in F_{n+1}(T)$ we say

$$
\theta \text { is consistent with } p \quad \text { if } \quad(\exists y) \theta(\bar{x}, y) \in p(\bar{x}) .
$$

(Since $T$ is a complete theory and $p(\bar{x})$ is a maximal consistent subset of $F_{n}(T)$ we have either $(\exists y) \theta(\bar{x}, y) \in p(\bar{x})$ or $\neg(\exists y) \theta(\bar{x}, y) \in p(\bar{x})$. Hence, this exactly expresses the consistency of $\theta$ and $p$.)

By Theorem 2.8, the isomorphism type of a homogeneous model $\mathcal{A}$ of a complete theory $T$ is determined entirely by its type spectrum $\mathbb{T}(\mathcal{A})$. To build a homogeneous model $\mathcal{A}$ of $T$ of a particular (Turing) degree we must first understand how to build a homogeneous model $\mathcal{A}$ whose type spectrum is a given class $\mathcal{C} \subseteq S(T)$. For any homogeneous model $\mathcal{A}$ of $T$ the type spectrum $\mathbb{T}(\mathcal{A})$ clearly satisfies the conditions (i) - (v) in Theorem 2.12. Goncharov [7] and Peretyat'kin [27] proved the converse.

Theorem 2.12 (Type Spectrum Theorem; Peretyat'kin [27], Goncharov [7]) Let T be a complete theory and $\mathcal{C} \subseteq S(T)$ be a countable set with $T \in \mathcal{C}$ satisfying conditions $(i)-(v)$ below. Then $T$ has a countable homogeneous model $\mathcal{A}$ with $\mathbb{T}(\mathcal{A})=\mathcal{C}$.

(i) Permutations

If $p\left(x_{0}, \ldots, x_{n-1}\right) \in \mathcal{C} \cap S_{n}(T)$ and $\pi$ is a permutation of $\{0, \ldots, n-1\}$ then $p\left(x_{\pi(0)}, \ldots, x_{\pi(n-1)}\right) \in \mathcal{C}$.

\section{(ii) Restriction}

If $p \in \mathcal{C} \cap S_{n}(T)$ and $m<n$ then $p_{<m} \in \mathcal{C}$ where $p_{<m}$ is the restriction of $p$ to formulas in which only $x_{i}, i<m$, appear free.

(iii) Disjoint Union

If $p, q \in \mathcal{C} \cap S_{n}(T)$ then there exists $r \in \mathcal{C} \cap S_{2 n}(T)$ such that

$$
r\left(x_{0}, \ldots, x_{2 n-1}\right) \supseteq p\left(x_{0}, \ldots, x_{n-1}\right) \cup q\left(x_{n}, \ldots, x_{2 n-1}\right) .
$$

(iv) Type Amalgamation Property (TAP)

If $p\left(\bar{x}, x_{n}\right), q\left(\bar{x}, x_{n}\right) \in \mathcal{C} \cap S_{n+1}(T)$ extend the same n-type $r(\bar{x}) \in \mathcal{C} \cap S_{n}(T)$ then there exists $s \in \mathcal{C} \cap S_{n+2}(T)$ such that

$$
s\left(\bar{x}, x_{n}, x_{n+1}\right) \supseteq p\left(\bar{x}, x_{n}\right) \cup q\left(\bar{x}, x_{n+1}\right) .
$$


(v) Extension Property (EP)

For every n-type $p(\bar{x}) \in \mathcal{C} \cap S_{n}(T)$ and $(n+1)$-ary $\theta\left(\bar{x}, x_{n}\right) \in F_{n+1}(T)$ consistent with $p(\bar{x})$ (as in (4)) there exists $q \in \mathcal{C} \cap S_{n+1}(T)$ such that

$$
p(\bar{x}) \cup\left\{\theta\left(\bar{x}, x_{n}\right)\right\} \subseteq q\left(\bar{x}, x_{n}\right) .
$$

\section{Decidable Copies of a Homogeneous Model}

By the 1970s Morley and others had explored the computable content of the Vaughtian models $\mathcal{A}$ of a complete theory $T$.

3.1 Morley's four properties Morley ([25], p. 236) noted the following four possible effectiveness properties of a model $\mathcal{A}$ of $T$ arranging in decreasing order of strength.

P1. There is a decidable model $\mathcal{A}$.

$\mathrm{P} 2$. There is a uniformly computable listing of $\mathbb{T}(\mathcal{A})$.

P3. The types in $\mathbb{T}(\mathcal{A})$ are all computable; that is, $\mathbb{T}(\mathcal{A})$ satisfies TAC.

P4. The theory $T$ is complete and decidable (CD).

Proposition 3.1 (Morley [25]) Morley noted the obvious implications

$$
\mathrm{P} 1 \Longrightarrow \mathrm{P} 2 \Longrightarrow \mathrm{P} 3 \Longrightarrow \mathrm{P} 4 \text {. }
$$

Remark 3.2 Morley ([25], p. 236) stated P1 - P4 and the above implications only for the case of the saturated model $\mathcal{A}$ so that $\mathbb{}(\mathcal{A})=S(T)$.

In the same paper Morley noted that the last two implications could not be reversed.

\section{Theorem 3.3 (Morley [25])}

(i) $\mathrm{P} 4 \Rightarrow \mathrm{P} 3$.

(ii) $\mathrm{P} 3 \not \mathrm{P} 2$.

There are now many examples of (i) and (ii) in the literature as we discuss later.

3.2 Presenting types for a complete decidable theory From now on we assume that $T$ is a complete decidable (CD) theory in a computable language $\mathcal{L}$. We now extend and sharpen the noneffective definitions for formulas and types presented in Section 2.2 for the computable case.

\section{Proposition 3.4}

(i) If $T$ is a complete decidable $(C D)$ theory then $F_{n}(T)$ and more precisely the tree $\mathcal{T}_{n}(T)$ are both decidable.

(ii) If $T$ is a CD theory, $n>1$, and $p$ is a computable (n-1)-type then $F_{n}(T) / p$ and the tree $\mathcal{T}_{n}(T) / p$ are computable where these are defined to be the formulas of $F_{n}(T)$ which are consistent with $p$.

Proof (i) Since $T$ is complete a formula, $\theta(\bar{x}) \in F_{n}(\mathcal{L})$ is consistent with $T$ if and only if $(\exists \bar{x}) \theta(\bar{x}) \in T$. Therefore, since $T$ is decidable $F_{n}(T)$ is also decidable.

(ii) We can do the same as in (i) but now we use the computability of both $T$ and $p$ to test the projections for consistency with a given formula $\theta(\bar{x}) \in F_{n}(T)$. 


\section{Definition 3.5}

(i) Given the fixed CD theory $T$ let $\left\{\theta_{i}\right\}_{i \in \omega}$ be an effective numbering of $F(T)=$ $\cup_{n} F_{n}(T)$.

(ii) For every $n>0$ and $n$-type $p$ we may assume $p$ decides every $k$-ary formula $\theta(\bar{x})$ for every $k<n$ as follows. Define

$$
\theta^{\prime}\left(x_{0}, \ldots, x_{n-1}\right)=\theta(\bar{x}) \wedge\left(\wedge_{j<n}\left(x_{j}=x_{j}\right)\right) .
$$

Add $\theta$ to $p$ just if $\theta^{\prime} \in p$ already. Now associate with $p$ a function $f \in 2^{\omega}$ such that $f(i)=1$ if and only if $\theta_{i} \in p$. Hence, every type corresponds to a function over all formulas $\theta_{i} \in F(T)$ but, clearly, $f_{p}(j)=0$ if $\theta_{j}$ is a $k$-ary formula for $k>n$.

(iii) Let $p$ be an $n$-type and $q$ be a $k$-type for $k<n$. Then $p$ and $q$ are inconsistent if and only if there exists a $k$-ary formula $\theta_{i}(\bar{x})$ such that $f_{p}(i) \neq f_{q}(i)$, and are consistent otherwise. If $p$ and $q$ are computable types then their consistency is a $\Pi_{1}$ condition.

(iv) For any type $p \in S(T)$ define $p\left\lceil s=p \cap\left\{\theta_{i}\right\}_{i<s}\right.$. Identify $p\lceil s$ with the function $f_{p}\left\lceil s\right.$ where $f_{p}(i)=1$ if and only if $\theta_{i} \in p$.

\subsection{Morley's Question on decidable copies}

Definition 3.6 Let $\mathcal{C} \subseteq S(T)$ be a set of types of a CD theory $T$ satisfying the five conditions of the Type Spectrum Theorem 2.12. If there exists some uniformly computable listing of $\mathcal{C}, X=\left\{p_{j}\right\}_{j \in \omega}$, then we call $X$ a $\mathbf{0}$-basis for $\mathcal{C}$. (Later we generalize this to a $\mathbf{d}$-basis for degrees $\mathbf{d}>\mathbf{0}$ in Section 5.1.)

Morley [25] rapidly turned attention to the remaining implication of whether P2 implies P1, and Morley ([25], p. 239) posed the following key question which is central to this paper.

Question 3.7 (Morley's Question) If $T$ is a complete decidable $(C D)$ theory and $\mathcal{A}$ is a homogeneous model of $T$ with a $\mathbf{0}$-basis $X$ for $\mathbb{T}(\mathcal{A})$, does $\mathcal{A}$ have a decidable copy $\mathcal{B}$ ? By the Homogeneous Uniqueness Theorem 2.8, this is equivalent to finding a decidable homogeneous model $\mathscr{B}$ of $T$ with $\mathbb{T}(\mathcal{A})=\mathbb{T}(\mathscr{B})$.

Morley had evidence to support a positive answer since he knew it to be true for the prime model case, and he himself had proved it for the saturated model case. Both of these are priority arguments which we shall recast in terms of the effective extension function for homogeneous models.

\subsection{The decidable prime criterion}

Definition 3.8 Let $T$ be a CD theory.

(i) A set $\mathcal{C} \subseteq S(T)$ is dense if $(\forall \theta \in F(T))(\exists p \in \mathcal{C})[\theta \in p]$.

(ii) An effective selector function for a dense set $\mathcal{C} \subseteq S(T)$ is a partial computable function $\varphi$ such that for every $i$ if $\theta_{i}(\bar{x}) \in F_{n}(T)$, then $\varphi(i)$ is defined and is the index of the characteristic function of some computable type $p \in S_{n}(T)$ which includes $\theta$.

Therefore, $\varphi$ is an effective algorithm to witness that $\mathcal{C}$ meets every basic open set $\mathcal{U}_{\theta}$ of $F_{n}(T)$ (i.e., such that $\theta \in F_{n}(T)$ ) by effectively producing a type in $\mathcal{C}$ in $u_{\theta}$. 
Theorem 3.9 (Decidable Prime Criterion; Harrington [9], Goncharov-Nurtazin [8]) Let $T$ be a complete atomic decidable (CAD) theory. Then the following are equivalent.

(i) T has a decidable prime model.

(ii) There is a uniform enumeration of $S^{P}(T)$, the principal types of $T$.

(iii) There is an effective selector function $f$ for $S^{P}(T)$.

Proof The implication (i) $\Longrightarrow$ (ii) is immediate. The reverse implication (ii) $\Longrightarrow$ (i) is the main content and is proved later in Theorem 4.10. For later purposes it is useful to reflect on the easy equivalence of (ii) and (iii).

(ii) $\Longrightarrow$ (iii). Let $\left\{p_{j}\right\}_{j \in \omega}$ be an effective listing of $S^{P}(T)$. Given $\theta_{i} \in F_{n}(T)$ let $f(i)$ be the least $j$ such that $p_{j} \in S_{n}^{P}(T)$ and $\theta_{i} \in p_{j}$.

(iii) $\Longrightarrow$ (ii). Fix $f$. Every principal type $p_{j} \in S_{n}^{P}(T)$ contains some atom $\theta_{i} \in F_{n}(T)$, but being an atom $\theta_{i}$ has exactly one extension to some $p \in S_{n}(T)$, that is, $p_{j}$ itself. Hence, $p_{f(i)}=p_{j}$ and $\left\{p_{f(i)}\right\}_{\theta_{i} \in F(T)}$ uniformly enumerates $S^{P}(T)$ because $\left\{\theta_{i}\right\}_{i \in \omega}$ uniformly enumerates $F(T)$.

Remark 3.10 Note that for every CD theory $T$ we have an effective selector function for $\mathcal{C}=S(T)$. If $\theta_{i} \in F_{n}(T)$, then $\theta_{i}$ is consistent and hence extendible on the computable tree $\mathcal{T}_{n}(T)$ to an infinite path. Indeed the lexicographically least path $q_{i}$ extending $\theta_{i}$ will do and will be a computable path. This gives us a uniformly effective procedure of passing from any $\theta_{i} \in \mathcal{T}_{n}(T)$ to the computable characteristic function of a type $q_{i}$ extending it. However, $F(T)$ is countable so the set of types $Q=\left\{q_{i}\right\}_{i \in \omega}$ obtained as the range of this procedure is also countable and may not be nearly all of $S(T)$. (Think of the theory of dense linear orderings with the countable set of rationals named. Every $\theta \in \mathcal{T}_{n}(T)$ can be extended to a path, but not all types will be obtained this way.) Even if $S(T)$ is countable, it is possible that $Q \neq S(T)$.

The point is that in the atomic case we necessarily obtain $Q=S(T)$ because for every type $p \in S^{P}(T)$ there is a $\theta_{i}$ which isolated it. This means that under the procedure above the only possible path $q_{i}$ extending $\theta_{i}$ is $p$ itself. Therefore, in Theorem 3.9, the proofs of (i) $\Longrightarrow$ (ii) and (ii) $\Longrightarrow$ (iii) hold for any model of a CD theory $T$, but (iii) $\Longrightarrow$ (ii) holds only for an atomic model. Millar's theorem in [24] produced a CD + TAC theory $T$ for which there is no computable enumeration of $S(T)$ and hence no decidable saturated model. Hence, the analogue of (iii) in Theorem 3.9 holds for $S(T)$ in place of $S^{P}(T)$ but (i) and (ii) fail. Later we shall make other remarks about selector functions.

For saturated models we need to uniformly list $S(T)$ not $S^{P}(T)$.

Theorem 3.11 (Decidable Saturated Criterion; Morley [25], Millar [24]) Let T be a complete decidable $(C D)$ theory with types all computable (TAC). The following are equivalent.

(i) Thas a decidable saturated model.

(ii) There is a uniformly computable enumeration of $S(T)$.

The main content of both theorems is the implication (ii) $\Longrightarrow$ (i) which we shall prove later in Theorem 4.11 using the effective extension property. Both involve a 
priority argument which arises during the effective construction for (i) because inconsistencies between types reveal themselves in a $\Sigma_{1}$ manner during the construction and we must change our target for a type $p \in S^{n}(T)$ to realize $\left\langle c_{0}, \ldots, c_{n-1}\right\rangle$. The problem is to arrange that this change (injury) occurs at most finitely often. In the prime and saturated cases, condition (ii) suffices to guarantee convergence, but in the full homogeneous case we need more.

\section{EEF and MEF Functions for a 0-Basis $X$}

Convention 4.1 For this section let $\mathcal{A}$ be a homogeneous model of a CD theory $T$ with $\mathbf{0}$-basis $X=\left\{p_{j}\right\}_{j \in \omega}$ for $\mathbb{T}(\mathcal{A})$, and fix the notation of Section 3.2.

Both Goncharov [7] and Peretyat'kin [27] negatively answered Morley's Question 3.7 by proving that $\mathcal{A}$ does not always have a decidable copy (as we discuss in Theorem 6.1 in Section 6.1). However, they showed that $\mathcal{A}$ does have a decidable copy $\mathcal{A}$ if there is a $\mathbf{0}$-basis $X$ with an additional assumption known as the effective extension property (EEP), an effectivization of the previous (noneffective) Extension Property (EP) (i.e., condition (v) of the Type Spectrum Theorem 2.12).

First note that since the $\mathbf{0}$-basis $X$ is uniformly computable it automatically has an effective selector function of Definition 3.8 as in the proof of (ii) $\Longrightarrow$ (iii) of the Decidable Prime Criterion 3.9 because given $\theta \in F_{n+1}(T)$ we can uniformly list the set $Y^{\theta}$ of all types $q \in X \cap S_{n+1}(T)$ which contain $\theta$ and choose the first such type. However, to effectivize the EP of Theorem 2.12 (v) we must now choose $q$ extending a given $n$-type $r \in S_{n}(T)$ which is consistent with $\theta$. We can still restrict our search to the uniformly computable set $Y^{\theta}$ (which eliminates any further dependence on $\theta$ ). However, both types $r$ and $q$ are infinite sets (albeit computable) and the question of whether they are inconsistent is merely a $\Sigma_{1}$ property (not necessarily computable). The following EEP property and its EEF function ensure that this search is computable, not only $\Delta_{2}$. This is the source of finite injury in constructing a decidable model in the Decidable Prime Criterion 3.9 or the Decidable Saturated Criterion 3.11.

\subsection{An effective extension function (EEF) for $\mathbb{T}(\mathcal{A})$}

Definition 4.2 (Effective Extension Function (EEF)) Let $\mathcal{A}$ be a homogeneous model of a complete decidable (CD) theory $T$ whose type spectrum $\mathbb{T}(\mathcal{A})$ has a $\mathbf{0}$-basis $X=\left\{p_{i}\right\}_{i \in \omega}$.

(i) A function $f$ is an extension function $(E F)$ for $X$ if, for every $n$,

(a) for every $n$-type $p_{i}(\bar{x}) \in X \cap S_{n}(T)$

(b) and every $(n+1)$-ary $\theta_{j}\left(\bar{x}, x_{n}\right) \in F_{n+1}(T)$ consistent with $p_{i}(\bar{x})$

the $(n+1)$-type $p_{f(i, j)} \in X \cap S_{n+1}(T)$ extends both; that is,

$$
p_{i}(\bar{x}) \cup\left\{\theta_{j}\left(\bar{x}, x_{n}\right)\right\} \subseteq p_{f(i, j)}\left(\bar{x}, x_{n}\right) .
$$

(Note that the EF function defined here is exactly that used in the Extension Property (EP), condition (v) of Theorem 2.12.)

(ii) If $f$ is also computable then $f$ is an effective extension function (EEF).

Theorem 4.3 (EEP Theorem; Goncharov [7], Peretyat'kin [27]) Let $\mathcal{A}$ be a homogeneous model of a complete decidable $(C D)$ theory $T$. Then $A$ has a decidable copy if and only if the type spectrum $\mathbb{T}(\mathcal{A})$ has a $\mathbf{0}$-basis $X=\left\{p_{i}\right\}_{i \in \omega}$ with an effective 
extension function (EEF). If so, we say that $X$ has the effective extension property (EEP) via $f$.

Goncharov [7] and independently Peretyat'kin [27] gave proofs of the EEP Theorem 4.3.

4.2 Monotone approximations to an EEF Morley posed his Question 3.7 because he knew the answer to be positive for certain special homogeneous models such as prime or saturated models. Now in the hindsight of the EEP Theorem 4.3 we know that if a prime or saturated model has a $\mathbf{0}$-basis $X$ then it has one with an EEF. It is not immediately obvious how to show this. There is a missing intermediate step of producing a monotone extension function $(M E F)$, that is, a $\Delta_{2}^{0}$ function which, given the same inputs as the EEF, converges to the same answer after a finite number of effective approximations. This concept, introduced by Goncharov ([7], p. 247) under the name of "extending function," is very useful both for the positive direction where we show that a given homogeneous model $\mathcal{A}$ with EEP such as a prime or saturated one does have a decidable copy and also in Section 5 where Lange proves various relativizations in the positive direction. The MEF function is also important for the negative direction in Section 6 of constructing a homogeneous model $\mathcal{A}$ and a $\mathbf{0}$-basis $X$ for $\mathbb{T}(\mathcal{A})$ such that $\mathcal{A}$ does not have a decidable copy, and it is crucial for negative results in Lange, Section 6. Let $\theta_{j}$ and $p_{i}\lceil k$ be as in Section 3.2. We now rearrange the MEF concept in a series of lemmas to present it more intuitively.

\subsection{Monotone functions on a $\mathbf{0}$-basis $X$}

Definition 4.4 (Monotone Function on $X$ ) $\quad$ A function $f(i)$ on $X$ is a monotone function on $X$ if there is a computable function $\widehat{f}(i, s)$ such that

(i) $f(i)=\lim _{s} \widehat{f}(i, s)$, and

(ii) $p_{\widehat{f}(i, s)}\left\lceil s \subseteq p_{\widehat{f}(i, s+1)} \uparrow(s+1)\right.$.

In this case we write $f(i)=\operatorname{mlim}_{s} \widehat{f}(i, s)$.

The idea of a monotone function is that we are building a computable type $q=\cup_{s} p_{\widehat{f}(i, j, s)} \backslash s$ as the union of a monotone sequence $\left\{p_{\widehat{f}(i, j, s)}\lceil s\}\right.$. At each stage $s$ we add to the characteristic function $q$ the initial segment $p_{\widehat{f}(i, j, s)}\lceil s$. Since we cannot later reverse that decision we need the monotonicity condition (ii) to guarantee that the only types considered after stage $s$ are those extending $p_{\widehat{f}(i, j, s)}\lceil s$.

By adjusting $X$ if necessary we may assume that every type $p_{i} \in X$ occurs infinitely often in $X$. Therefore, we shall assume that $\widehat{f}$ is monotonic in the sense of (ii) and also the usual sense that $\widehat{f}(i, s) \leq \widehat{f}(i, s+1)$.

Lemma 4.5 (Equality Lemma) If $X=\left\{p_{i}\right\}_{i \in \omega}$ and $Y=\left\{q_{i}\right\}_{i \in \omega}$ are $\mathbf{0}$-bases for $\mathbb{T}(\mathcal{A})$ there is a monotonic function $g$ on $X$ such that $p_{g(i)}=q_{i}$.

Proof Define $\widehat{g}(i, s)=(\mu k>i)\left[p_{k}\left\lceil s=q_{i}\lceil s]\right.\right.$. Now there is some $p_{k} \in X$, $k>i$, such that $p_{k}=q_{j}$ and $g(i)=\operatorname{mlim}_{s} \widehat{g}(i, s)$ is the least such $k$. 


\subsection{A monotone extension function (MEF) for $X$}

Definition 4.6 Let $T$ be a CD theory with 0 -basis $X=\left\{p_{i}\right\}_{i \in \omega}$. A function $f(i, j)$ on $X$ is a monotone extension function (MEF) for $X$ if for every $n$-type $p_{i} \in X$ and $(n+1)$-ary formula $\theta_{j}$ consistent with $p_{i}$ the following hold.

(i) The function $f(i, j)$ is an extension function for $X$, as in Definition 4.2 (i).

(ii) There is a computable function $\widehat{f}$ such that $f(i, j)=\operatorname{mlim}_{s} \widehat{f}(i, j, s)$ as in Definition 4.4.

(iii) $p_{\widehat{f}(i, j, s)}\left\lceil s\right.$ is consistent with $p_{i}$.

Lemma 4.7 (MEF Transfer Lemma) If $X=\left\{p_{i}\right\}_{i \in \omega}$ and $Y=\left\{q_{i}\right\}_{i \in \omega}$ are $\mathbf{0}$-bases for $\mathbb{T}(\mathcal{A})$ and $X$ has an MEF function $f(i, j)$ then $Y$ also has an MEF function.

Proof Let $f(i, j)=\operatorname{mlim}_{s} \widehat{f}(i, j, s)$ be an MEF for $X$. Fix an $n$-type $q_{i} \in Y$ and an $(n+1)$-ary formula $\theta_{j}$ consistent with $q_{i}$. By the Equality Lemma 4.5 there is a monotonic function $g(i)=\operatorname{mlim}_{s} g(i, s)$ such that $p_{g(i)}=q_{i}$. Define computable functions $\widehat{J}(i, j, s)$ and $\widehat{F}(i, j, s)$ simultaneously by induction on $s$.

Stage $s=0$. Define $\widehat{J}(i, j, 0)=j$ and $\widehat{F}(i, j, 0)=\widehat{f}(\widehat{g}(i, 0), j, 0)$.

Stage $s+1$. Define

$$
\begin{gathered}
\widehat{J}(i, j, s+1)= \begin{cases}\widehat{J}(i, j, s) & \text { if } \widehat{g}(i, s+1)=\widehat{g}(i, s), \\
(\mu k)\left[\theta_{k}=p_{\widehat{F}(i, j, s)\lceil s}\right] & \text { otherwise. }\end{cases} \\
\widehat{F}(i, j, s+1)=\widehat{f}(\widehat{g}(i, s+1), \widehat{J}(i, j, s+1), s+1) .
\end{gathered}
$$

(The point is that the sequence $\{\widehat{f}(i, j, s)\}$ is guaranteed to produce the amalgamator only if the original inputs $i$ and $j$ remain fixed. Therefore, whenever $\widehat{g}(i, s) \neq \widehat{g}(i, s+1)$ we must restart the $\widehat{f}$ function on a new pair of inputs and for monotonicity we must give as $\theta^{\prime}$ the conjunction of all $\theta_{k} \in p_{\widehat{F}(i, j, s)}$ for $k \leq s$ since we have already put these in the type $p_{F(i, j)}$ being constructed.)

Now choose $t$ such that $g(i)=\widehat{g}(i, s)$ for all $s \geq t$. Then $\widehat{J}(i, j, t)=\widehat{J}(i, j, s)$ for all $s \geq t$. For all stages $s>t$ we compute

$$
\widehat{F}(i, j, s)=\widehat{f}\left(i^{\prime}, j^{\prime}, s\right)
$$

with $i^{\prime}=g(i)$ and $j^{\prime}=\operatorname{mlim}_{s} \widehat{J}(i, j, s)$. Hence, $F(i, j)=\operatorname{mlim}_{s} \widehat{F}(i, j, s)$ converges monotonically to some $m$ by the monotonicity of $\widehat{f}$.

Finally, define

$$
\widehat{H}(i, j, s)=(\mu k)\left[p_{\widehat{F}(i, j, s)}\left\lceil s \subseteq q_{k}\right] .\right.
$$

Now $X=Y$ so there is some $q_{k} \in Y$ such that $q_{k}=p_{m}$. Hence $H(i, j)=\operatorname{mlim}_{s} \widehat{H}(i, j, s)$ produces the $Y$ amalgamator for $n$-type $p_{i}$ and formula $\theta_{j}$. 
4.5 The MEF theorem The following characterization corresponds to Goncharov [7], Corollary 4.

Theorem 4.8 (MEF Theorem) Let $\mathcal{A}$ be a homogeneous model of a $C D$ theory $T$ whose type spectrum $\mathbb{T}(\mathcal{A})$ has a $\mathbf{0}$-basis. Then the following are equivalent.

(i) Some $\mathbf{0}$-basis for $\mathbb{T}(\mathcal{A})$ has an EEF.

(ii) Some $\mathbf{0}$-basis for $\mathbb{T}(\mathcal{A})$ has an $M E F$.

(iii) Every $\mathbf{0}$-basis for $\mathbb{T}(\mathcal{A})$ has an $M E F$.

Proof Clearly $(i) \Longrightarrow(i i)$ and $(i i i) \Longrightarrow(i i)$.

(ii) $\Longrightarrow$ (iii). Apply the Transfer Lemma 4.7.

(ii) $\Longrightarrow(i)$. Fix a $\mathbf{0}$-basis $X=\left\{p_{j}\right\}_{j \in \omega}$ with $\operatorname{MEF} f(i, j)=\operatorname{mlim}_{s} \widehat{f}(i, j, s)$. Define another 0-basis $Y=\left\{q_{j}\right\}_{j \in \omega}$ with an EEF as follows. First define $q_{2 j}=p_{j}$ so that $X \subseteq Y$. Next given $\theta_{i}$ and $q_{j}$ we find an amalgamator $p_{k}$ as follows and copy $p_{k}$ on row $q_{2\langle i, j\rangle+1}$. Hence, $X=Y$.

Define $g(j)=\operatorname{mlim}_{s} \widehat{g}(j, s)$ as in the Equality Lemma 4.5. Define $\psi_{s}=$ $p_{\widehat{f}(i, \widehat{g}(j, s), s)} \mid s$ and define $q_{2\langle i, j\rangle+1}=\cup_{s} \psi_{s}$.

Theorem 4.9 (Full EEP Theorem) Let $\mathcal{A}$ be a homogeneous model of a CD theory $T$ whose type spectrum $\mathbb{T}(\mathcal{A})$ has a $\mathbf{0}$-basis. Then the following are equivalent.

(i) A has a decidable copy.

(ii) Some $\mathbf{0}$-basis for $\mathbb{T}(\mathcal{A})$ has an EEF.

(iii) Some 0-basis for $\mathbb{T}(\mathcal{A})$ has an MEF.

(iv) Every $\mathbf{0}$-basis for $\mathbb{T}(\mathcal{A})$ has an $M E F$.

Proof Combine the MEF Theorem 4.8 and the EEP Theorem 4.3.

4.6 Finding an MEF for a prime model The proof of the main implication (ii) $\Longrightarrow$ (i) of the Decidable Prime Criterion 3.9 was originally and still remains a priority argument, but we can easily derive it from the EEP Theorem (which also employs a finite injury proof). To show that a prime model $\mathcal{A}$ has a decidable copy it suffices by the Full EEP Theorem 4.9 to show that $\mathbb{T}(\mathcal{A})$ has a $\mathbf{0}$-basis with an MEF function.

Theorem 4.10 (MEF for Decidable Prime Models) Let $\mathcal{A}$ be a prime model of a complete atomic decidable $(C A D)$ theory $T$ such that $\mathbb{T}(\mathcal{A})=S^{P}(T)$ has a $\mathbf{0}$-basis $X$. Then $X$ has an $M E F$, and hence $\mathcal{A}$ has a decidable copy.

Proof Given an $n$-type $p_{i}$ and $(n+1)$-ary formula $\theta_{j}$ consistent with $p_{i}$ define

$$
\widehat{f}(i, j, 0)=(\mu k>i)\left[\theta_{j} \in p_{k} \in X \cap S_{(n+1)}(T)\right] .
$$

Let $\widehat{f}(i, j, s+1)$ be the least $k>i$ such that

1. $\theta_{j} \in p_{k} \in X \cap S_{(n+1)}(T)$,

2. $p_{k} \uparrow s \supseteq p_{\widehat{f}(i, j, s)} \uparrow s$, and

3. $p_{k} \uparrow s+1$ is consistent with $p_{i}$.

Now $p_{i}$ is a principal type and hence contains an atom $\alpha$. Eventually there will be a large enough stage $t$ so that $p_{\widehat{f}(i, j, t)}$ also contains $\alpha$. Hence, for all $s \geq t$, we have $\widehat{f}(i, j, s)=\widehat{f}(i, j, t)$. 
4.7 Finding an MEF for a saturated model The same proof applies to the saturated model case, but with a different reason for convergence.

Theorem 4.11 (MEF for Decidable Saturated Models; Morley [25], Millar [24]) Let $\mathcal{A}$ be a saturated model of a complete decidable $(C D)$ theory $T$ with types all computable (TAC) whose type spectrum $\mathbb{T}(\mathcal{A})$ has a $\mathbf{0}$-basis. Then $X$ has an $M E F$, and hence A has a decidable copy.

Proof Define $\widehat{f}(i, j, s)$ as in the prime model MEF Theorem 4.10. Now $p_{m}=$ $\cup_{s} p_{\widehat{f}(i, j, s)}\left\lceil s\right.$ is a type in $S_{(n+1)}(T)$ consistent with $p_{i}$. But $\mathbb{T}(\mathcal{A})=S(T)$ includes all types consistent with $T$ so $f(i, j)=\operatorname{mlim}_{s} \widehat{f}(i, j, s)=m$.

\section{Positive Results on Homogeneous Models}

The Full EEP Theorem 4.9 completely characterized when a homogeneous model $\mathcal{A}$ has a decidable copy just as the Decidable Prime Criterion 3.9 completely characterized when a prime model $\mathcal{A}$ has a decidable copy. For prime models this was the starting point for a variety of results about the degrees of copies of $\mathcal{A}$ as developed in Csima [3] and Csima, Hirschfeldt, Knight, and Soare [5], and elsewhere. Analogously we now consider the degrees of copies of a homogeneous model.

5.1 A d-basis for the degree spectrum $X=\mathbb{T}(\mathcal{A}) \quad$ In the following definition we still require that all types in $X=\left\{p_{j}\right\}_{j \in \omega}$ be computable (TAC), but we weaken the condition that there be a uniformly computable listing (0-basis) for them. This allows us to consider a wider class of models, for example, prime models of complete atomic decidable theories.

Definition 5.1 Let $\mathcal{A}$ be a homogeneous model of a complete decidable (CD) theory $T$ whose types $X=\left\{p_{j}\right\}_{j \in \omega}$ are all computable (TAC). Let $\mathbf{d}$ be a (Turing) degree. Then $X$ is a d-basis for $\mathbb{T}(\mathcal{A})$ if there exists a function $g \leq \mathbf{d}$ such that $g(j)$ is a $\Delta_{0}$-index for $p_{j}$.

We view $g$ as defining a matrix with rows $\left\{p_{j}\right\}_{j \in \omega}$. It is not sufficient to specify a d-computable function $h(i, j)$ which can uniformly compute the matrix, that is, $h(i, j)=p_{j}(i)$. Rather $g(j)$ must specify an index $e=g(j)$ for the computable characteristic function of $p_{j}$ from which Turing machine with index $e$ can actually compute $p_{j}$ with no further help from any oracle. (In the TAC case it is easy to confuse $g$ and the weaker $h$. This confusion case has caused several errors in the literature.)

5.2 Relativizing the EEF and MEF to a degree d The proof of the EEP Theorem 4.3 and MEF Theorem 4.8 can be relativized in the usual way to any degree $\mathbf{d}$. We usually do not use the full power of this relativization because we know $T$ is a complete decidable theory and $\mathcal{A}$ has some kind of basis. For example, in Lange's Low Basis Theorem 5.3 below we are given a homogeneous model $\mathcal{A}$ of a $\mathrm{CD}$ theory $T$ and a $\mathbf{0}^{\prime}$-basis $X$ for $\mathbb{T}(\mathcal{A})$. Lange builds a new basis $Y$ for $\mathbb{T}(\mathcal{A})$ and simultaneously a low degree $\mathbf{d}$ and function $f \leq \mathbf{d}$ such that (1) $\mathbf{d}$ uniformly computes $Y$ and (2) $f$ is an extension function for $Y$. The relativization of the Full EEP Theorem 4.9 to $\mathbf{d}$ we use here is the following. 
Theorem 5.2 (d-EEP Theorem) Let $\mathcal{A}$ be a homogeneous model of a $C D$ theory $T$ with type spectrum $\mathbb{T}(\mathcal{A})$ and suppose there is $\mathbf{d}$-computable function $g(x, y)$ such that $\left\{g_{y}\right\}_{y \in \omega}=\mathbb{T}(\mathcal{A})$, where $g_{y}=\lambda x[g(x, y)]$ viewed as a "row" in the matrix $g(x, y)$. Then the following are equivalent.

(i) A has a d-decidable copy.

(ii) Some d-computable listing of $\mathbb{T}(\mathcal{A})$ has a $\mathbf{d}$-computable EEF.

(iii) Some d-computable listing of $\mathbb{1}(\mathcal{A})$ has a $\mathbf{d}$-computable $M E F$.

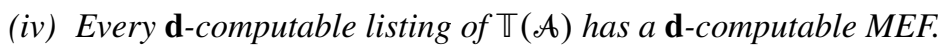

Proof The proof is the same as that of Theorem 4.9 but relativized to $\mathbf{d}$.

In Theorem 5.2 we avoided the term $\mathbf{d}$-basis because that refers to a matrix which is $\mathbf{d}$-uniformly computable but also whose rows are computable, with $\Delta_{0}$-indices uniformly computable from $\mathbf{d}$, for example, a $\mathbf{0}^{\prime}$-basis. Here we have in mind only the most simpleminded approach. Consider the case of a $\mathbf{0}$-basis where everything is computable, including the matrix which is uniformly computable. Relativize that idea to $\mathbf{d}$.

There are cases where the input is of this form, for example, in Lange's Theorem 5.3 where the prime model $\mathcal{A}$ admits a $\mathbf{0}^{\prime}$-basis for $\mathbb{T}(\mathcal{A})$. However, Lange's output there is a copy $\mathscr{B}$ of $\mathcal{A}$ which has low degree d. Although the rows of the basis for $\mathscr{B}$ happen to be computable because they are the rows of the $\mathbf{0}^{\prime}$-basis for $\mathcal{A}$, Lange does not claim that this basis for $\mathscr{B}$ constitutes a $\mathbf{d}$-basis. To constitute a $\mathbf{d}$-basis, $\mathbf{d}$ would have to uniformly compute the $\Delta_{0}$-indices of the rows in this basis. It suffices here to apply the relativized Theorem 5.2: $\mathbf{d}$ can uniformly compute a $\Delta_{0}^{\mathbf{d}}$ index for every row, and with respect to these indices, there are $\mathbf{d}$-computable functions which are the EEF and MEF.

The partial relativization of the Full EEP Theorem 4.9 to d we use here says if a homogeneous model $\mathcal{A}$ of a complete decidable theory $T$ has a basis uniformly computable in $\mathbf{d}$ with a d-effective extension function then $\mathcal{A}$ has a d-decidable copy. Applying this to $f$ and $Y$, we obtain a d-decidable copy of $\mathcal{A}$.

In cases where we assume $\mathcal{A}$ has a $\mathbf{0}$-basis $X$, we show $X$ has a $\mathbf{d}$-monotone extension function $f$ to show $\mathcal{A}$ has a d-decidable copy. As a $\mathbf{0}$-basis $X$ can also be viewed as a basis uniformly computable in $\mathbf{d}$, a partial relativization of the Full EEP Theorem gives that $X$ with $f$ implies the existence of a d-decidable copy. Similarly, to show a homogeneous $\mathcal{A}$ with a $\mathbf{0}$-basis $X$ has no $\mathbf{d}$-decidable copy, it suffices to show that $X$ has no d-monotone extension function.

5.3 Two approaches to homogeneous models There are two main approaches to studying the computable content of homogeneous models of a CD theory. One approach is to fix a homogeneous model which satisfies reasonable computability conditions and study its degree spectrum, that is, the degrees of its isomorphic copies. This general idea will be the subject of this section, Section 5, with positive results and the next section, Section 6, on negative results. The second approach is to study which degrees are strong enough to effectively find a homogeneous model for any CD theory. This will be the subject of Section 7.

The Full EEP Theorem 4.9 exactly characterized when a homogeneous model of a CD theory has a decidable copy. Notably, if some $\mathcal{A} \models T$ is to have any hope of 
having a decidable copy, $\mathcal{A}$ must have a $\mathbf{0}$-basis. Thus, following our first approach, we assume we are given a complete decidable theory $T$ and a homogeneous model $\mathcal{A}$ of $T$ with a d-basis. Then we study the degree spectrum of (the elementary diagram of) $\mathcal{A}$.

5.4 Low copies of homogeneous models As we shall see in Theorem 6.1, Millar, Peretyat'kin, and Goncharov gave a negative answer to Morley's Question 3.7 and showed that $\mathbf{0}$ is not strong enough to compute an isomorphic copy for every homogeneous $\mathcal{A}$ with a $\mathbf{0}$-basis. On the other hand, it is easy to see using the Full EEP Theorem 4.9 relativized to $\mathbf{0}^{\prime}$ that any homogeneous $\mathcal{A}$ with even a $\mathbf{0}^{\prime}$-basis has a $\mathbf{0}^{\prime}$-decidable copy.

Hence $\mathbf{0}^{\prime}$ can always decide an isomorphic copy of such a homogeneous model, but $\mathbf{0}$ may not suffice, which is the same pattern as for prime and saturated models. The first remark follows by noticing that a $\mathbf{0}^{\prime}$-oracle can compute a $\mathbf{0}^{\prime}$-basis $X$ and a $\mathbf{0}^{\prime}$ extension function $f$ for $X$ by using a $\mathbf{0}^{\prime}$-oracle to answer $\Sigma_{1}$ questions about the consistency of the types in $X$. The second remark follows by Theorem 6.1.

Next we consider intermediate degrees $\mathbf{d}, \mathbf{0}<\mathbf{d}<\mathbf{0}^{\prime}$, as possible degrees of isomorphic copies of a homogeneous $\mathcal{A}$ which has a $\mathbf{0}$-basis. The first result about intermediate degrees gives that every homogeneous model $\mathcal{A}$ with a $\mathbf{0}^{\prime}$-basis has an isomorphic copy decidable in a low degree.

Theorem 5.3 (Homogeneous Low Basis Theorem; Lange [18]) Let $T$ be a complete decidable theory and $\mathcal{A} \models T$ be a homogeneous model with a $\mathbf{0}^{\prime}$-basis $X=\mathbb{T}(\mathcal{A})$. Then there is a low degree $\mathbf{d}$ and a $\mathbf{d}$-decidable copy of $\mathcal{A}$.

The proof involves a $\mathbf{0}^{\prime}$-construction of a low degree $\mathbf{d}$, a basis $Y$ which $\mathbf{d}$ uniformly computes, and a computable function which is an extension function for $Y$. The forcing conditions involve a finite number of infinite computable rows of $Y$ whose $\Delta_{0}$ indices have been computed uniformly in $\mathbf{0}^{\prime}$ together with a finite number of other elements in the matrix $Y$. We guarantee $\mathbf{d}=\operatorname{deg}(Y)$ is low by simultaneously forcing the jump $\{e\}^{Y}(e)$ as we build $Y$. The proof requires that $\mathbf{0}^{\prime}$ knows all the computable indices for the types $p_{j} \in X$ so that a $\mathbf{0}^{\prime}$ oracle suffices to do the forcing. The theorem gives the following analogous result for prime models as a corollary.

Corollary 5.4 (Prime Low Basis Theorem; Csima [3]) Let $T$ be a complete atomic decidable theory with a prime model $\mathcal{A}$. Then there exists a low degree $\mathbf{d}$ and a d-decidable prime model $B$ of $T$.

Proof Since $\mathcal{A}$ is prime, $\mathbb{T}(\mathcal{A})=S^{P}(T)$, the principal types of $T$. By the Homogeneous Low Basis Theorem 5.3 relativized to $\mathbf{0}^{\prime}$ and the Homogeneous Uniqueness Theorem 2.8 it suffices to show that $\mathcal{A}$ has a $\mathbf{0}^{\prime}$-basis. First note that every $p_{j} \in X$ is computable because it contains an atom $\alpha_{j}$ and $S(T)$ contains only one type extending $\alpha_{j}$. Second, $\boldsymbol{0}^{\prime}$ can uniformly list all the atoms of $\cup_{n} F_{n}(T)$ and every atom effectively determines an isolated type. Being an atom is a $\Pi_{1}$-property because $\alpha$ is not an atom if and only if there exist incompatible extensions $\beta$ and $\gamma$ of $\alpha$, which is a $\Sigma_{1}$-property.

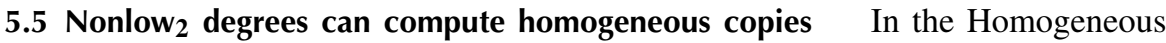
Low Basis Theorem 5.3 we fixed a homogeneous model $\mathscr{A}$ with a d-basis and asked which degrees can compute a copy of that specific $\mathcal{A}$. Now we attempt to find degrees 
d which are strong enough to compute a d presentation of any nontrivial homogeneous model with a $\mathbf{0}$-basis. This idea gives rise to the following definition.

Definition 5.5 Degree $\mathbf{d}$ is $\mathbf{0}$-bounding if for any nontrivial homogeneous model $\mathcal{A}$ with a $\mathbf{0}$-basis, there exists a d-decidable $\mathcal{B} \cong \mathcal{A}$.

Csima, Hirschfeldt, Knight, and Soare ([5], [3]) studied the prime bounding degrees, that is, the degrees which given any complete atomic decidable theory $T$ can compute a prime model in $T$. They showed that, within the $\Delta_{2}^{0}$-degrees, the prime bounding degrees are exactly the nonlow 2 degrees. The above definition is one way to extend the notion of bounding to the homogeneous case. Another possible extension will be discussed in Section 8.

Theorem 5.6 (0-Bounding Theorem, Lange [18]) If $\mathbf{d} \leq \mathbf{0}^{\prime}$ is nonlow 2 (i.e., $\left.\mathbf{d}^{\prime \prime}>\mathbf{0}^{\prime \prime}\right)$ then $\mathbf{d}$ is 0-bounding.

Let $\mathbf{d} \leq \mathbf{0}^{\prime}$ be nonlow 2 . Lange proves there exists a $\mathbf{d}$-MEF for every $\mathbf{0}$-basis $X=\left\{p_{j}\right\}_{j \in \omega}$ of a homogeneous $\mathcal{A}$. Then the Full EEP Theorem 4.9 implies that $\mathcal{A}$ has a d-decidable copy. To show that $X$ has a d-MEF, we need to be able to dapproximate whether a given type $p_{k}$ is an amalgamator of $p_{i}$ and $\theta_{j}$. In general, determining whether $p_{k}$ is an amalgamator of $p_{i}$ and $\theta_{j}$ is a $\mathbf{0}^{\prime}$ question, because finding an inconsistency between two computable types $p_{k}$ and $p_{m}$ is a $\Sigma_{1}^{0}$ statement.

By a characterization of nonlow $2 \Delta_{2}^{0}$ degrees (see Soare [30], Chapter 4) given a $\mathbf{0}^{\prime}$-computable function $g$, there exists a $\mathbf{d}$-computable function $f$ which infinitely often escapes (is greater than) $g$. We can use a d-computable escape function for a carefully chosen $\mathbf{0}^{\prime}$-function which looks for inconsistencies to build the desired d-MEF.

5.6 If $S(T)$ satisfies TAC we obtain all nonzero degrees In this section we return to studying the degrees of copies of a specific homogeneous $\mathcal{A}$ with a $\mathbf{0}$-basis. If the conditions about the theory $T$ are strengthened, we can obtain a more complete picture of these degrees. Since we assume $\mathcal{A}$ has a $\mathbf{0}$-basis, all the types realized in $\mathcal{A}$ must be computable. However, the types outside of $\mathbb{T}(\mathcal{A})$, that is, the types $p \in S(T)-\mathbb{T}(\mathcal{A})$, may very well not be computable. Thus, we can add computability conditions on these "outside" types in $S(T)$ to get stronger results. Specifically, we restrict our study to theories $T$ in which all types in $S(T)$ are computable and to homogeneous models $\mathcal{A}$ with $\mathbf{0}$-bases.

Theorem 5.7 (Goncharov [7], Millar [22]) Let $T$ be a complete decidable theory with $S(T)$ uniformly computable. If $\mathcal{A}$ is a homogeneous model with a $\mathbf{0}$-basis, then A has a decidable copy.

If $S(T)$ consists of only computable types but is not uniformly computable, we still get the following strong result.

Theorem 5.8 (Full Basis Theorem; Lange [18]) Let $T$ be a complete decidable theory with $S(T)$ consisting of computable types. Let $\mathcal{A}$ be a homogeneous model of $T$ with a $\mathbf{0}$-basis. Then for every nonzero degree $\mathbf{d}$ there is a model $\mathscr{B} \cong \mathcal{A}$ with elementary diagram $D^{e}(\mathscr{B})$ of degree $\mathbf{d}$.

A key idea of this theorem (as observed by Harris) is that if $S(T)$ consists of all computable types, an amalgamator $p_{k}$ for a given type $p_{i}$ and formula can be chosen 
to be principal over $p_{i}$. More formally, there exists a formula $\psi(\bar{x}, y)$ consistent with $p_{i}(\bar{x})$ such that

$$
p_{k}=\left\{\theta(\bar{x}, y):(\forall y)(\psi(\bar{x}, y) \rightarrow \theta(\bar{x}, y)) \in p_{i}(\bar{x})\right\} .
$$

Then the theorem follows by applying Hirschfeldt's technique [12] to prove the analogous result for prime models.

\section{Negative Results on Homogeneous Models}

The MEF characterization and Full EEP Theorem 4.3 can also be used to give negative results about homogeneous models. First we sketch how to prove the following negative result by diagonalizing against possible MEF functions.

6.1 A counterexample to Morley's question Goncharov, Millar, and Peretyat'kin gave counterexamples that negatively answered Morley's Question 3.7. Each example consisted of a homogeneous model $\mathcal{A}$ with a $\mathbf{0}$-basis $X$ but no decidable copy.

Theorem 6.1 (Goncharov [7], Peretyat'kin [27], Millar [23]) There is a homogeneous model $\mathcal{A}$ of a complete decidable theory $T$ such that $\mathbb{T}(\mathcal{A})$ has a $\mathbf{0}$-basis but no decidable copy.

The MEF portion of the Full EEP Theorem 4.9(iv) gives a more perspicuous way to prove this theorem. We simply need to construct a homogeneous model $\mathcal{A}$ with a $\mathbf{0}$ basis $X$ which has no MEF. (If we try to diagonalize against the EEF characterization in Theorem 4.9(ii), then we would need to ensure that no basis $Y$ for $\mathcal{A}$ has an EEF. Here we construct $X$ and we consider only one such $X$.)

We shall build a homogeneous model $\mathcal{A}$ and a $\mathbf{0}$-basis $X$ for $\mathbb{T}(\mathcal{A})$ with no $\mathbf{0}$-MEF by diagonalizing against every possible $\operatorname{MEF} \varphi_{e}$. This requires both an understanding of which $\mathbf{0}$-bases are the type spectrum $\mathbb{T}(\mathcal{A})$ for a homogeneous $\mathcal{A}$ and a strategy to defeat MEFs. Theorem 2.12 says that a given $\mathbf{0}$-basis $Y$ equals $\mathbb{T}(\mathcal{A})$ of a homogeneous $\mathcal{A}$ if and only if $Y$ is closed under some basic model theoretic properties such as closure under permutations and type amalgamation. Hence we must satisfy the following positive requirement:

$P: \quad X$ satisfies the homogeneity closure conditions in Theorem 2.12 .

To ensure $X$ is a $\mathbf{0}$-basis, we build $X$ computably in stages so that each row corresponds to a type in a simple and flexible CD theory $T$. We satisfy $P$ by assigning each homogeneity closure condition a marker $H$. Then at the beginning of the construction, $H$ is placed on an empty row of $X$. Throughout the construction, $H$ can only be moved to another row finitely often, and the row on which $H$ comes to rest is built to satisfy the corresponding closure condition.

Now we develop a strategy to defeat $\varphi_{e}$ as an MEF. If $f$ is an MEF for $X$, then for any $n$-type $p_{i}$ in $X$ and for any $(n+1)$-ary formula $\theta_{j}$ consistent with $p_{i}$, $\Lambda_{s}=\hat{f}(i, j, s)$ must monotonically trace out a true amalgamator for $p_{i}$ and $\theta_{j}$ as described in Definition 4.6. In particular, $\Lambda=\lim _{s} \Lambda_{s}$ exists, and $p_{k}$ amalgamates $p_{i}$ and $\theta_{j}$. Thus, to ensure $\varphi_{e}$ is not an MEF, we monitor the behavior of $\varphi_{e}$ on some specified 1-type $p_{i_{e}}$ and 2-ary formula $\theta_{j_{e}}$ which are consistent. That is, we ensure that marker $\Lambda_{e, s}=\varphi_{e}\left(i_{e}, j_{e}, s\right)$ is never able to remain on any row if it is tracing out a true amalgamator to $p_{i_{e}}$ and $\theta_{j_{e}}$. To show $\varphi_{e}$ is not an MEF, it suffices to show for all $k$ : 
$N_{e, k}:$ If $\Lambda_{e}$ is describing a true amalgamator, then $\Lambda_{e}$ does not come to rest on row $k$.

The challenge in the construction of the desired $\mathbf{0}$-basis $X$ is to simultaneously satisfy $P$ and $N_{e, k}$ for all $e$ and $k$. In particular, requirement $P$ requires the existence of a true amalgamator for $p_{i_{e}}$ and $\theta_{j_{e}}$ in $X$, but $N_{e, k}$ requires that $\Lambda_{e}$ cannot find it.

6.2 A generalized counterexample and characterization Millar had produced a complete atomic decidable theory which had no decidable (or even computable) prime model. Csima, Hirschfeldt, Knight, and Soare ([5], [3]) extended this by constructing such a theory with no prime model of low 2 degree.

We now do the corresponding result for homogeneous models. In the preceding subsection we sketched a proof of Theorem 6.1 that there is a CD theory $T$ and a model $\mathcal{A}$ of $T$ with a $\mathbf{0}$-basis but no decidable copy. The next theorem shows that we can avoid any low 2 copies as well. Although this is an analogue of the result for prime models ([5], [3]), the proof here is much harder, mainly because of the difficulty in meeting the five conditions of Theorem 2.12 to make $\mathcal{A}$ homogeneous.

Theorem 6.2 (Generalized Counterexample; Lange [17]) Let $\mathbf{d}$ be a low $\Delta_{2}^{0}$ degree. There exists a homogeneous $A$ with a $\mathbf{0}$-basis such that $\mathcal{A}$ has no $\mathbf{d}$-decidable copy.

The idea of the proof is that if $\mathbf{d} \leq \mathbf{0}^{\prime}$ is low low $_{2}$, then there exists a $\mathbf{0}^{\prime}$-computable listing of all the $\mathbf{d}$-computable functions. Then, we can defeat all $\mathbf{d}$-MEFs for the basis $X$ by diagonalizing against a computable approximation to this listing of the d-computable functions. This theorem then completes the characterization of $\Delta_{2}^{0}$ 0-bounding degrees.

Theorem 6.3 (Characterization of 0-Bounding; Lange [17]) A degree $\mathbf{d} \leq \mathbf{0}^{\prime}$ is $\mathbf{0}$-bounding if and only if $\mathbf{d}$ is nonlow 2 , that is, $\mathbf{d}^{\prime \prime}>\mathbf{0}^{\prime \prime}$.

Proof By Theorem 5.6 and Theorem 6.2.

\section{Homogeneous Bounding Degrees}

In the results so far we have fixed a particular CD theory $T$ and a homogeneous model $\mathcal{A}$ of $T$ and explored the possible degrees of isomorphic copies of $\mathcal{A}$. Another approach is to ask whether a given degree $\mathbf{d}$ can compute some homogeneous model for every $\mathrm{CD}$ theory. We call a degree $\mathbf{d}$ homogeneous bounding if every $\mathrm{CD}$ theory has a d-decidable homogeneous model.

Recall a degree $\mathbf{d}$ is a Peano Arithmetic (PA) degree if $\mathbf{d}$ is the degree of a complete extension of the first-order theory of Peano Arithmetic. The next result says that although the prime bounding degrees are defined in terms of jumps ([5], [3]), the homogeneous bounding degrees are more related to PA degrees and computing paths through (nonextendible) trees.

Theorem 7.1 (Csima, Harizanov, Hirschfeldt, Soare [4]) A degree $\mathbf{d}$ is homogeneous bounding if and only if $\mathbf{d}$ is a PA degree.

Before sketching the idea of the proof, we need to review well-known properties of PA degrees. 
Theorem 7.2 The following are equivalent for any Turing degree $\mathbf{d}$.

1. The d-computable sets form a basis for $\Pi_{1}^{0}$. (That is, every infinite computable binary tree has an infinite $\mathbf{d}$-computable path.)

2. If $U$ and $V$ are disjoint c.e. sets, then there is a $\mathbf{d}$-computable set $S$ such that $U \subseteq S$ and $V \cap S=\varnothing$. (Such a set $S$ is called $a$ separating set for $U$ and $V$.)

3. The degree $\mathbf{d}$ is the degree of a complete extension of PA.

4. The degree $\mathbf{d}$ is the degree of the elementary diagram $D^{e}(\mathcal{A})$ of a nonstandard model $\mathcal{A}$ of $P$ A.

One way to build a homogeneous model of a given theory is via an elementary chain (as in Marker [20]). This argument repeatedly uses Lindenbaum's Lemma (that every consistent set of sentences can be extended to a complete theory) which is equivalent to (1) above. Lindenbaum's Lemma can be carried out effectively in a degree d (in the sense that every consistent computable set of sentences can be extended to a complete decidable theory) if and only if $\mathbf{d}$ is a PA degree. This gives half of Theorem 7.1.

For the other half of Theorem 7.1, Csima, Harizanov, Hirschfeldt, and Soare construct a particular CD theory $T$ such that every homogeneous model of $T$ has a PA degree. The language of $T$ has infinitely many unary predicate symbols $\left\{P_{i}\right\}_{i \in \omega}$, infinitely many binary predicate symbols $\left\{R_{i}\right\}_{i \in \omega}$, a unary predicate symbol $D$, and a binary predicate symbol $E$.

Let $U$ be the set of Gödel numbers of sentences provable from PA and let $V$ be the set of Gödel numbers of sentences refutable from PA. By Theorem 7.2, any degree that can compute a separating set for $U$ and $V$ is a PA degree. The idea of the proof is to define the theory $T$ in such a way that

1. if $\mathcal{A} \vDash T$ then $E^{\mathcal{A}}$ is an equivalence relation, and if $c, d \in A$ are in different $E^{\mathcal{A}}$-equivalence classes and satisfy exactly the same $P_{i}$, then the set $\left\{i: R_{i}^{\mathcal{A}}(c, d)\right\}$ is a separating set for $U$ and $V$; and

2. every homogeneous model of $T$ must contain such elements $c$ and $d$.

These conditions imply that if $\mathcal{A} \vDash T$ is homogeneous, then the atomic diagram of $\mathcal{A}$ can compute a separating set for $U$ and $V$, and hence has a PA degree.

\section{Conclusion}

There are a number of related results on the degrees of Vaughtian models.

8.1 Separating properties from bounding prime models Csima, Hirschfeldt, Knight, and Soare [5] formulated conditions on a set $A$ sufficient to guarantee that every complete atomic decidable (CAD) theory $T$ has a prime model whose elementary diagram is computable in $A$. Such a set $A$ or its degree is called prime bounding. Unexpectedly, there emerged eight other apparently unrelated properties of $A$ which turned out to be equivalent to the prime bounding property for those sets $A$ which are $\Delta_{2}^{0}$ (i.e., $A \leq_{\mathrm{T}} \varnothing^{\prime}$ ). These included degree properties (such as nonlow 2 ), computability properties (such as escaping every $\varnothing^{\prime}$ function), topological properties (such as meeting a sequence of $\Delta_{2}$ open sets), and a monotonic property which has been used by Shore and others to study models of $\aleph_{1}$-categorical theories.

Conidis, at citation [2], examined these nine properties (P0)-(P8) and observed that although they are all equivalent for $A \leq_{\mathrm{T}} \varnothing^{\prime}$ they are not necessarily equivalent in the general case $A \nless_{\mathrm{T}} \varnothing^{\prime}$. Conidis has shown that they fall into three groups, 
each group consisting of logically equivalent properties. This gives the complete classification of these properties in the general case and it sheds light on the general mathematical relationships among these familiar and important properties. Most of these properties arise in other areas of mathematics.

For example, two of the most important properties are ( $\mathrm{P} 0)$, the escape property, and ( $\mathrm{P} 3)$, the isolated path property, which is equivalent to the prime bounding property. It is easy to prove that (P0) implies (P3). It is not at all clear how to prove the converse, but that is just what Conidis has done.

(P0) The escape property $\left(\forall g \leq_{\mathrm{T}} 0^{\prime}\right)\left(\exists f \leq_{\mathrm{T}} A\right)\left(\exists^{\infty} x\right)[g(x) \leq f(x)]$, where " $\left(\exists^{\infty}\right)$ " denotes "there exist infinitely many".

(P3) The isolated path property For every computable tree $\mathcal{T} \subseteq 2^{<\omega}$ with no terminal nodes and with isolated paths dense,

$$
\left(\exists g \leq_{\mathrm{T}} A\right)(\forall \theta \in \mathcal{T})\left[g_{\theta} \in\left[\widetilde{\mathcal{T}}_{\theta}\right] \& g_{\theta} \text { is isolated }\right] .
$$

The implication of (P3) to (P0) has a very interesting and unexpected proof. To show the inequivalence of nonlow 2 with other properties $(\mathrm{P} i)$, Conidis uses a $\varnothing^{\prime \prime}$ forcing argument to force the double jump $A^{\prime \prime}$ while coding in whatever function is necessary to make ( $\mathrm{P} i$ ) hold of $A$ while (P0) ( $A$ nonlow $_{2}$ ) fails since $\varnothing^{\prime \prime}$ can compute $A^{\prime \prime}$ from the construction.

8.2 Computably enumerable degrees of prime models A second interesting area is a finer study of degrees of certain models. The papers by Csima [3] and Csima, Hirschfeldt, Knight, and Soare [5] studied $\Delta_{2}^{0}$ degrees, and to some extent degrees in general, of prime models of a CAD theory $T$. In other areas of computability it has been fruitful to study the computably enumerable (c.e.) degrees. The elementary diagram $\mathcal{A}$ of the model cannot itself be a c.e. set else the provables and refutables of $T$ are c.e. and $T$ is computable. However, we can ask when we have a model $\mathcal{A}$ whose elementary diagram has c.e. degree. Epstein [6] has improved some results by Csima [3] as follows.

Theorem 8.1 (Epstein, Density of Prime C.E. Low Degrees) Let $\mathcal{T}$ be a computable tree with no terminal nodes and isolated paths dense. Let $C$ be c.e. and nonlow 2 , and let $D$ be c.e. and low, $D \leq_{\mathrm{T}} C$. Then there is a low c.e. set $\widehat{B}$ such that $D<_{\mathrm{T}} \widehat{B}<_{\mathrm{T}} C$ and $\widehat{B}$ computes a listing of the isolated paths of $\mathcal{T}$.

Corollary 8.2 (Epstein, Prime Low C.E. Basis Theorem) Let $T$ be a complete atomic decidable theory. Then there is a prime model of $T$ with low c.e. degree.

Theorem 8.3 (Epstein, Prime Low C.E. Minimal Pair) Let T be a complete atomic decidable theory. Then there is a minimal pair of c.e. low degrees $\mathbf{b}$ and $\mathbf{c}$ such that $\mathbf{b}$ and $\mathbf{c}$ are degrees of prime models of $T$.

Theorem 8.4 (Epstein, Homogeneous C.E. Low Basis Theorem) Let A be a homogeneous model with a $\mathbf{0}$-basis. That is, there is a computable function $g$ such that $\left\{\varphi_{g(e)}\right\}_{e \in \omega}$ is a listing of the types in A. Then A has an isomorphic copy of low c.e. degree.

8.3 C.E. degrees of saturated models Harris [10] calls a degree d saturated bounding if for any complete, decidable (CD) theory with types all computable (TAC) there is a saturated model whose elementary diagram is computable in $\mathbf{d}$. Harris gives negative results on c.e. degrees which complement the previous positive 
results. He first [11] develops a characterization of $\operatorname{low}_{n}$ sets for all $n$ in terms of escape functions. He shows that if the degree is also c.e. then in addition to the escape property for a $\operatorname{low}_{n}$ degree there is also an alignment property. He uses these two simultaneously to prove the following theorem [10].

Theorem 8.5 (Harris) Let $\mathbf{d}$ be a lown c.e. degree for any $n \geq 1$. Then there is a complete decidable theory with all types computable which has no $\mathbf{d}$-decidable saturated model.

\section{References}

[1] Baldwin, J. T., and A. H. Lachlan, "On strongly minimal sets," The Journal of Symbolic Logic, vol. 36 (1971), pp. 79-96. Zbl 0217.30402. MR 0286642. 151

[2] Conidis, C., "Separating properties from prime models," in preparation. 145, 166

[3] Csima, B. F., "Degree spectra of prime models," The Journal of Symbolic Logic, vol. 69 (2004), pp. 430-42. Zbl 1069.03025. MR 2058182. 144, 146, 160, 162, 163, 165, 167

[4] Csima, B. F., V. S. Harizanov, D. R. Hirschfeldt, and R. I. Soare, "Bounding homogeneous models," forthcoming in The Journal of Symbolic Logic, 2007. 165

[5] Csima, B. F., D. R. Hirschfeldt, J. F. Knight, and R. I. Soare, "Bounding prime models," The Journal of Symbolic Logic, vol. 69 (2004), pp. 1117-42. Zbl 1071.03021. MR 2135658. 145, 160, 163, 165, 166, 167

[6] Epstein, R., "Computably enumerable degrees of Vaught's models," in preparation, 2006. 145,167

[7] Goncharov, S. S., "Strong constructivizability of homogeneous models," Algebra $i$ Logika, vol. 17 (1978), pp. 363-88, 490. English translation, Algebra and Logic, vol. 17 (1979), pp. 247-63. Zbl 0441.03015. MR 538302. 144, 145, 146, 152, 156, 157, 159, 163,164

[8] Goncharov, S. S., and A. T. Nurtazin, "Constructive models of complete decidable theories," Algebra i Logika, vol. 12 (1973), pp. 125-42, 243. English translation, Algebra and Logic, pp. 67-77. Zbl 0278.02038. MR 0398816. 144, 155

[9] Harrington, L., "Recursively presentable prime models," The Journal of Symbolic Logic, vol. 39 (1974), pp. 305-9. Zbl 0332.02055. MR 0351804. 144, 155

[10] Harris, K., "Bounding saturated models," in preparation. 145, 167, 168

[11] Harris, K., “A characterization of the low $_{n}$ degrees below $\mathbf{0}^{\prime}$," in preparation. 145, 168

[12] Hirschfeldt, D. R., "Computable trees, prime models, and relative decidability," Proceedings of the American Mathematical Society, vol. 134 (2006), pp. 1495-98 (electronic). Zbl 1099.03024. MR 2199197. 144, 146, 164

[13] Jockusch, C. G., Jr., "Degrees in which the recursive sets are uniformly recursive," Canadian Journal of Mathematics, vol. 24 (1972), pp. 1092-99. Zbl 0221.02029. MR 0321716. 145

[14] Jónsson, B., "Homogeneous universal relational systems," Notices of the American Mathematical Society, vol. 5 (1958), p. 776. Zbl 0173.00505. MR 0125021. 147, 151 
[15] Jónsson, B., “Universal relational systems," Mathematica Scandinavica, vol. 4 (1956), pp. 193-208. Zbl 0077.25302. MR 0096608. 147

[16] Keisler, H. J., and M. D. Morley, "On the number of homogeneous models of a given power," Israel Journal of Mathematics, vol. 5 (1967), pp. 73-78. Zbl 0153.32103. MR 0223225. 150

[17] Lange, K., "A characterization of the $\mathbf{0}$-basis homogeneous bounding degrees," in preparation. $146,165,170$

[18] Lange, K., "The degree spectra of homogeneous models," in preparation. 146, 162, 163,170

[19] Macintyre, A., and D. Marker, "Degrees of recursively saturated models," Transactions of the American Mathematical Society, vol. 282 (1984), pp. 539-54. Zbl 0557.03046. MR 732105. 145

[20] Marker, D., Model Theory: An Introduction, vol. 217 of Graduate Texts in Mathematics, Springer-Verlag, New York, 2002. Zbl 1003.03034. MR 1924282. 144, 147, 150, 166

[21] Martin, D. A., "Classes of recursively enumerable sets and degrees of unsolvability," Zeitschrift für mathematische Logik und Grundlagen der Mathematik, vol. 12 (1966), pp. 295-310. Zbl 0181.30504. MR 0224469. 144

[22] Millar, T. S., "Type structure complexity and decidability," Transactions of the American Mathematical Society, vol. 271 (1982), pp. 73-81. MR 648078. 163

[23] Millar, T., "Homogeneous models and decidability," Pacific Journal of Mathematics, vol. 91 (1980), pp. 407-18. Zbl 0467.03007. MR 615688. 146, 164

[24] Millar, T. S., "Foundations of recursive model theory," Annals of Mathematical Logic, vol. 13 (1978), pp. 45-72. Zbl 0432.03018. MR 482430. 144, 155, 160

[25] Morley, M., “Decidable models,” Israel Journal of Mathematics, vol. 25 (1976), pp. 233-40. Zbl 0361.02067. MR 0457190. 144, 145, 153, 154, 155, 160

[26] Morley, M., and R. Vaught, "Homogeneous universal models," Mathematica Scandinavica, vol. 11 (1962), pp. 37-57. Zbl 0112.00603. MR 0150032. 147

[27] Peretyat'kin, M. G., “A criterion of strong constructivizability of a homogeneous model," Algebra i Logika, vol. 17 (1978), pp. 436-54, 491. English translation, Algebra and Logic, pp. 290-301. Zbl 0436.03030. MR 538306. 144, 145, 146, 152, 156, 157,164

[28] Post, E. L., "Recursively enumerable sets of positive integers and their decision problems," Bulletin of the American Mathematical Society, vol. 50 (1944), pp. 284-316. Reprinted in Davis (1965), pp. 304-37. Zbl 0063.06328. MR 0010514. 144

[29] Ryll-Nardzewski, C., "On the categoricity in power $\leq \aleph_{0}, "$ Bulletin de l'Académie Polonaise des Sciences. Série des Sciences Mathématiques, Astronomiques et Physiques, vol. 7 (1959), pp. 545-48 (unbound insert). Zbl 0117.01101. MR 0111681. 147

[30] Soare, R. I., Computability Theory and Applications, Springer-Verlag, Heidelberg. To appear. 147, 163

[31] Vaught, R. L., "Homogeneous universal models of complete theories," Abstract 550-29, Notices of the American Mathematical Society, vol. 5 (1958), p. 775. 147, 151 
[32] Vaught, R. L., "Prime models and saturated models," Abstract 550-39, Notices of the American Mathematical Society, vol. 5 (1958), p. 780. 147, 151

[33] Vaught, R. L., "Universal relational systems for elementary classes and types," Abstract 550-1, Notices of the American Mathematical Society, vol. 6 (1958), p. 671. Meeting of the American Mathematical Society, Claremont, California, November, 1958. 147, 151

[34] Vaught, R. L., "Denumerable models of complete theories," pp. 301-21 in Infinistic Methods (Proceedings of Symposium on Foundations of Mathematics, Warsaw, 1959), Pergamon Press, 1961. Zbl 0113.24302. MR 0186552. 143, 144, 147, 150, 151

\section{Acknowledgments}

The second author presented some of this material at the Vaught's Conjecture/ Classification Workshop at University of Notre Dame on Saturday, May 21, 2005. The first author presented some of these and other results at an A.M.S. Special Session on October 23, 2004 at Northwestern University and also at an A.M.S. meeting at Notre Dame, April 8-9, 2006. The material in Lange [18] and [17] is part of her Ph.D. dissertation for which Robert Soare and Denis Hirschfeldt are cosupervisers. The authors are grateful to Denis Hirschfeldt for many discussions and suggestions on these topics.

Department of Mathematics

University of Chicago

Chicago IL 60637-1546

klange@math.uchicago.edu

http://www.math.uchicago.edu/ klange/

soare@math.uchicago.edu

http://www.people.cs.uchicago.edu/ soare/ 